\title{
Role for RIP1 in mediating necroptosis in experimental intracerebral hemorrhage model both in vivo and in vitro
}

\author{
Haitao Shen ${ }^{1,2}$, Chenglin Liu ${ }^{1,2}$, Dongping Zhang ${ }^{1,2}$, Xiyang Yao ${ }^{1}$, Kai Zhang ${ }^{1}$, Haiying Li*,1 and Gang Chen ${ }^{\star, 1}$
}

Cell death is a hallmark of second brain injury after intracerebral hemorrhage (ICH); however, the mechanism still has not been fully illustrated. In this study, we explored whether necroptosis, a type of regulated necrosis, has an essential role in brain injury after ICH. We found that inhibiting receptor-interacting protein 1 (RIP1) - a core element of the necroptotic pathway - by a specific chemical inhibitor or genetic knockdown attenuated brain injury in a rat model of $\mathrm{ICH}$. Furthermore, necroptosis of cultured neurons could be induced by conditioned medium from microglia stimulated with oxygen hemoglobin, and this effect could be inhibited by TNF- $\alpha$ inhibitor, indicating that TNF- $\alpha$ secreted from activated microglia is an important factor in inducing necroptosis of neurons. Undoubtedly, overexpression of RIP1 increased conditioned medium-induced necroptosis in vitro, but this effect was partially diminished in mutation of serine kinase phosphorylation site of RIP1, showing that phosphorylation of RIP1 is the essential molecular mechanism of necroptosis, which was activated in the in vitro model of ICH. Collectively, our investigation identified that necroptosis is an important mechanism of cell death in brain injury after ICH, and inhibition of necroptosis may be a potential therapeutic intervention of $\mathrm{ICH}$.

Cell Death and Disease (2017) 8, e2641; doi:10.1038/cddis.2017.58; published online 2 March 2017

Intracerebral hemorrhage $(\mathrm{ICH})$ is the second largest type of stroke, accounting for $\sim 15 \%$ of all patients with stroke. ${ }^{1,2} \mathrm{ICH}$ is associated with fast progression, high mortality and high morbidity. It has been reported that, with mortality rates close to $40 \%$ in 1 month, patients have legacy paralysis, aphasia and other severe disabilities after $\mathrm{ICH}$. The incidence of $\mathrm{ICH}$ also increased significantly with the increase of population age. ${ }^{3}$ Primary brain injury after $\mathrm{ICH}$ is due to hematoma mass effect and mechanical damage to adjacent brain tissues, ${ }^{4}$ and the secondary brain injury is a key reason to cause nerve function damage in patients with $\mathrm{ICH}^{5,6}$ In secondary brain injury after $\mathrm{ICH}$, pathological changes, including cell death, cerebral edema and blood-brain barrier (BBB) damage, occur in brain tissues surrounding hematoma. ${ }^{7}$ Mechanism of secondary brain injury after $\mathrm{ICH}$ includes excitatory aminoacid toxicity, inflammatory response, expression of proteolytic enzyme and the toxic effects of hematoma release product. ${ }^{5,7}$ Cell death is an important factor in secondary brain injury after $\mathrm{ICH}$.

In recent years, necrosis as another form of cell death causes more attention. The definition of necrosis is based on its pathological morphological characteristics; it is a passive cell death mostly caused by overwhelming stress such as dramatic changes in temperature or $\mathrm{pH}$. Necrotic cells rapidly lose cell membrane integrity, and cell membrane swelling and mitochondrial dysfunction occur. The membrane rupture caused a large number of cytoplasmic components to leak from the cell and then induced inflammation in the surrounding tissues. ${ }^{8}$ Previous studies suggested that necrosis is an occasional and irregular event and is difficult to study. ${ }^{9}$ However, Yuan and co-workers ${ }^{10}$ reported, for the first time, necroptosis as a form of necrosis that can be regulated. ${ }^{10}$ Necroptosis has similar morphological characteristics (including early membrane integrity, cell and intracellular organelle swelling) with necrosis, but it is caspase-independent programmed cell death. ${ }^{11}$

Recent studies have shown that death receptors on the cell membrane mediated different pathways of cell death depending on the state of cell or local physiological or pathophysiological microenvironment; for example, in the event of TNF- $a$-induced cell death, TNF- $a$ and its receptors firstly formed a trimer, and then recruited proteins containing the death domain (DD) formed a complex, which activated the downstream apoptotic pathway. However, if caspase activity was inhibited, the cells would go to the necroptotic pathway. RIP1 was recruited to TNFR1 through its DD, which bound directly to the DD of TNFR1, and it was activated by multiple forms of ubiquitination. The activation of RIP1 led to the recruitment of RIP3, MLKL and caspase-8, forming a complex called 'necrosome' involved in necroptotic pathway. Thus, RIP1 played the role of adaptor in mediating necroptosis. Activation of RIP3 activated three key enzymes of cell metabolic pathways - glycogen phosphorylase, glutamine synthetase, and glutamate dehydrogenase - which caused overproduction of reactive oxygen species (ROS). ROS caused DNA damage, damage to mitochondrial membrane

\footnotetext{
${ }^{1}$ Department of Neurosurgery and Brain and Nerve Research Laboratory, The First Affiliated Hospital of Soochow University, Suzhou, Jiangsu Province, China

${ }^{*}$ Corresponding author: G Chen or H Li, Department of Neurosurgery and Brain and Nerve Research Laboratory, The First Affiliated Hospital of Soochow University, 188 Shizi Street, Suzhou 215006, China. Tel: +86 13771908806; Fax: +86 051267780165; E-mail: nju_neurosurgery@163.com or Tel:+86 15716201037; Fax:+86 0512 67787180; E-mail: 348022626@qq.com.

${ }^{2}$ These authors contributed equally to this work.

Received 27.10.16; revised 28.12.16; accepted 03.1.17; Edited by Y Shi
} 
permeability and lysosome damage, eventually leading to cell death. $^{11,12}$

Previous studies have confirmed that microglia was quickly activated in brain tissues after $\mathrm{ICH}$, and it secreted a large number of inflammatory factors, including the TNF- $a$, which can induce necroptosis. ${ }^{13}$ Under the stimulation of
TNF-a, a part of the brain cells undergo apoptosis and a part may undergo necroptosis in brain tissues after $\mathrm{ICH}$. Necrotic cell death is common in a wide variety of pathological conditions, including stroke. ${ }^{14}$ Compared with numerous investigations on the mechanisms of apoptotic cell death, fewer studies have explored necrotic cell death in $\mathrm{ICH}$. Here a

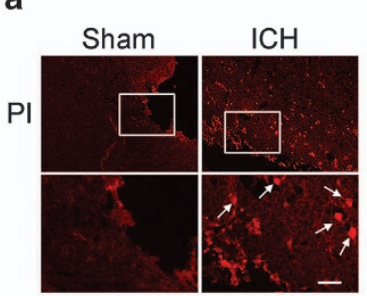

b

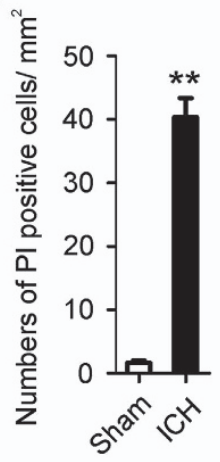

C

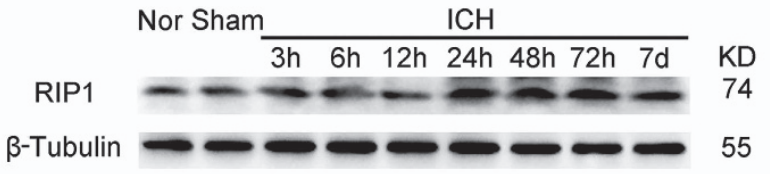

d

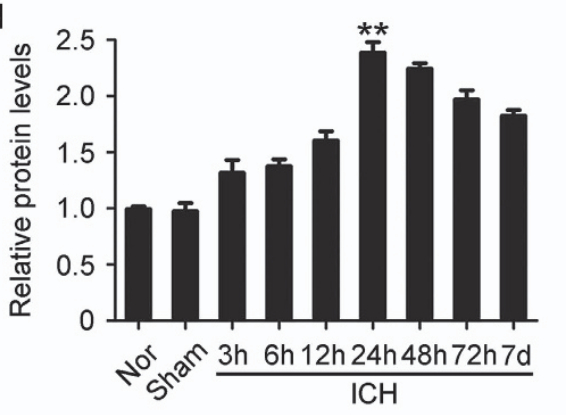

$\mathbf{f}$
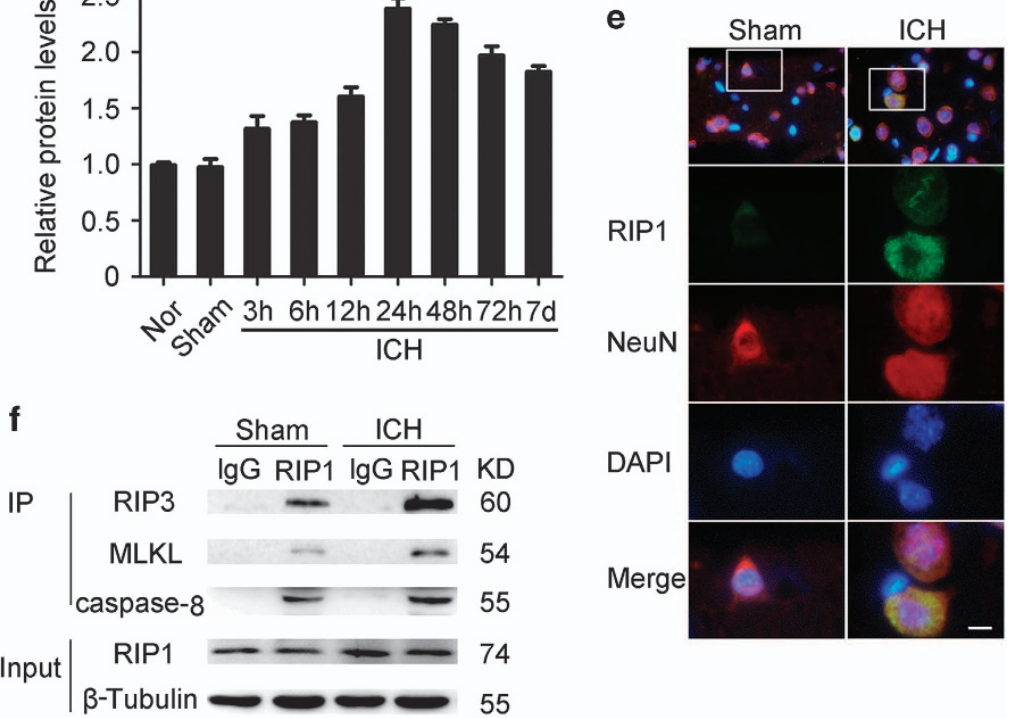

g
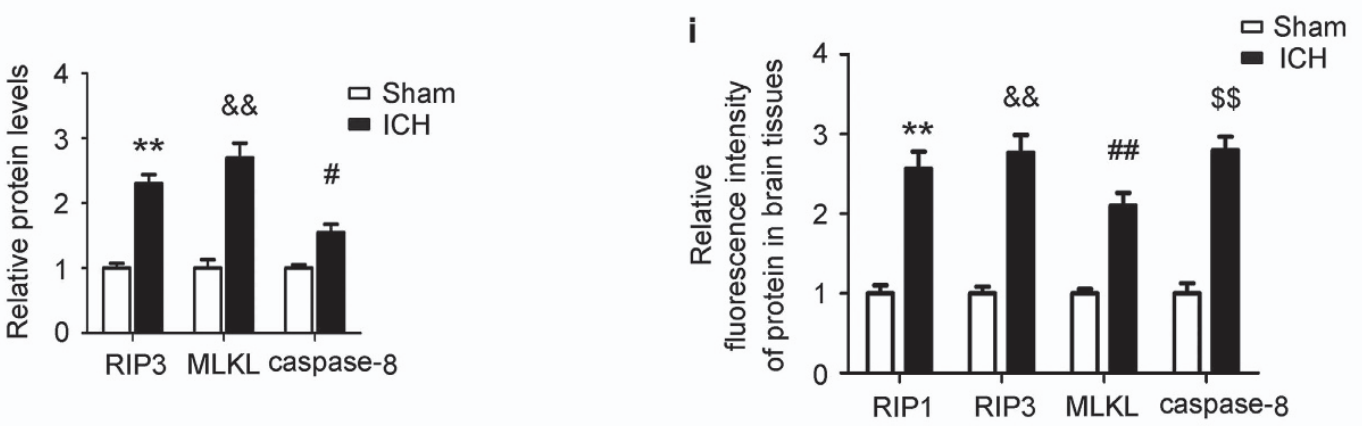

h

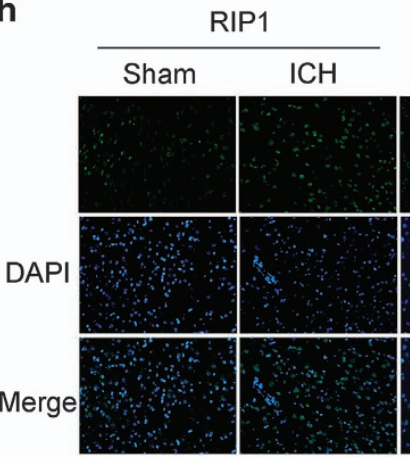

RIP3

Sham $\mathrm{ICH}$

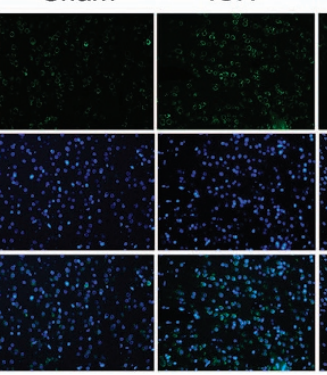

MLKL

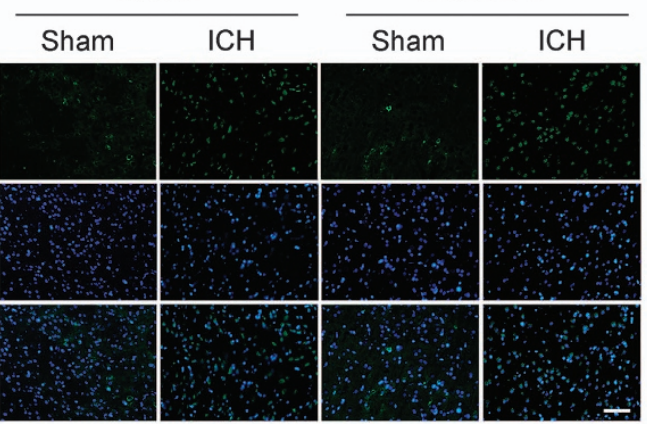


we studied the role of necroptosis, a type of regulated necrosis, in $\mathrm{ICH}$.

\section{Results}

Necroptosis was activated in brain tissues after ICH. To explore the involvement of necroptosis in brain tissues after $\mathrm{ICH}$, we detected propidium iodide-positive $(\mathrm{PI}+)$ cells in frozen brain sections at $24 \mathrm{~h}$ after $\mathrm{ICH}$. Notably, compared with that in the Sham group, the PI+ cells were remarkably increased in brain tissues surrounding hematomas after $\mathrm{ICH}$ $(P<0.0001, n=6$; Figures $1 \mathrm{a}$ and $\mathrm{b})$. We also determined the expression of RIP1, which is a major regulator for necroptosis in brain tissues; the results of western blot suggested that the expression levels of RIP1 were progressively upregulated and peaked at $24 \mathrm{~h}$ after $\mathrm{ICH}(P=0.0003$ versus Sham group, $n=3$; Figures $1 \mathrm{c}$ and $\mathrm{d}$ ). The results of immunofluorescent staining also showed that the expression level of RIP1 in neurons was remarkably increased at $24 \mathrm{~h}$ after $\mathrm{ICH}$ (Figure 1e).

We further evaluated the formation of necrosome, which is an important hallmark of activation of necroptosis in brain tissues after ICH; anti-RIP1 antibody was used by immunoprecipitation (IP), and RIP3, MLKL and caspase-8 were detected by immunoblotting. Increased interactions of RIP1 and RIP3, RIP1 and MLKL, and RIP1 and caspase- 8 were observed in brain tissues after $\mathrm{ICH}$ (all $P<0.01$ versus Sham group, $n=3$, Figures $1 \mathrm{f}$ and $\mathrm{g}$ ). It suggested that the formation of necrosome was significantly increased in brain tissues after $\mathrm{ICH}$ than that in the Sham group. The results of immunofluorescence also showed that expressions of these four proteins were significantly upregulated after $\mathrm{ICH}$ than that in the Sham group (all $P<0.01$ versus Sham group, $n=6$, Figures $1 \mathrm{~h}$ and i). These results indicated that necroptosis was activated in brain tissues after $\mathrm{ICH}$.

Inhibitor of necroptosis and apoptosis differentially attenuates brain injury after $\mathbf{I C H}$. To determine whether necroptosis contributes to brain injury after $\mathrm{ICH}$, the specific inhibitor of RIP1, necrostatin-1 (Nec-1), was used, and z-VAD, a caspase inhibitor, which can inhibit apoptosis, was also used as a positive control in a rat model of ICH. Nec-1 reduced the $\mathrm{PI}+$ cells but had no significant effect in TUNEL+ cells, whereas z-VAD only downregulated the TUNEL+ cells, and did not affect the $\mathrm{PI}+$ cells in brain tissues after $\mathrm{ICH}$. The combination of $z-V A D$ and Nec- 1 both obviously reduced the $\mathrm{PI}+$ cells and TUNEL+ cells (all $P<0.01, n=6$; Figures $2 \mathrm{a}$ and $b$ ). The results of IP also demonstrated that interactions of RIP1 and RIP3, RIP1 and MLKL, and RIP1 and caspase-8 were significantly inhibited by $\mathrm{Nec}-1$ but not by $\mathrm{z}-\mathrm{VAD}$ (all $P<0.05, n=3$; Figures $2 \mathrm{c}$ and d). These results indicated that necroptosis and apoptosis occurred in different cells at same times and were independent of each other in brain tissues after $\mathrm{ICH}$.

BBB permeability was assessed using albumin extravasation, and the western blot was used to test the albumin level in brain tissues; the results also suggested that treatment with $\mathrm{Nec}-1$ in $\mathrm{ICH}$ rats can improve albumin extravasation and BBB injury ( $P=0.0461, n=3$; Figures $2 \mathrm{e}$ and $\mathrm{f})$. Brain water content was calculated by the wet and dry weight method; the results showed that, compared with $\mathrm{ICH}$ group, the brain water content was significantly reduced in the $\mathrm{ICH}+\mathrm{Nec}-1$ group $(P=0.0109$ in Ipsi-CX and $P=0.0077$ in Ipsi-BG, $n=6$; Figure $2 \mathrm{~g}$ ). The neurological score of the $\mathrm{ICH}+\mathrm{Nec}-1$ group was significantly lower than that in the $\mathrm{ICH}$ group $(P=0.0094$, $n=6$; Table 1). The levels of TNF- $a$ in the cerebrospinal fluid (CSF) were measured by ELISA; the results confirmed that the level of TNF- $a$ was obviously reduced by Nec- 1 treatment, indicating that Nec-1 inhibits inflammation in $\mathrm{ICH}$ rats $(P<0.0001, n=6$; Figure $2 \mathrm{~h})$. These results suggested that necroptosis contributes to brain injury after $\mathrm{ICH}$, including neuronal dysfunction, brain water content, BBB permeability and inflammation.

\section{Blockade necroptosis by knockdown of RIP1 improves} brain injury after ICH. To further define the role of RIP1mediated necroptosis in neuronal dysfunction after $\mathrm{ICH}$, we used knockdown by small interfering RNA (siRNA) interference and overexpression by recombinant adenoviruses transfection of RIP1 as treatment in rat model of $\mathrm{ICH}$. Knockdown of RIP1 reduced the $\mathrm{PI}+$ cells, but overexpression of RIP1 increased the PI+ cells in brain tissues after ICH (both $P<0.0001, n=6$; Figures $3 \mathrm{a}$ and $\mathrm{b}$ ). The results of IP also demonstrated that interactions of RIP1 and RIP3, RIP1 and MLKL, and RIP1 and caspase-8 were significantly inhibited by knockdown of RIP1, but they were increased by overexpression of RIP1 (all $P<0.05, n=3$; Figures $3 \mathrm{c}$ and

\footnotetext{
Figure 1 Indicators of necroptosis were discovered in brain tissues after ICH. (a) The necroptosis of cells in brain tissues was detected by PI labeling. As shown, compared with the Sham group, considerable Pl+cells were detected in frozen sections of brain tissues in rats at $24 \mathrm{~h}$ after ICH. Arrows point to PI+ cells. Scale bar $=50 \mu \mathrm{m}$. (b) Related with (a), it revealed relative levels of PI+ cells, ${ }^{* *} P<0.0001$ versus Sham group, unpaired $t$-test, $n=6$. (c) The results of western blot suggested that the expression levels of RIP1, a major regulator for necroptosis, were obviously upregulated, and it reached peak at $24 \mathrm{~h}$ in brain tissues after ICH. (d) Related with (c), quantitative analysis of expression levels of RIP1 in brain tissues within 1 week after ICH. ${ }^{* *} P=0.0003$ versus Sham group, unpaired $t$-test, $n=3$. (e) Double immunofluorescence (IF) analysis was performed with antibodies for RIP1 (green) and NeuN (red). Nuclei were fluorescently labeled with DAPI (4',6-diamidino-2-phenylindole) (blue). Representative images of the Sham group and the $\mathrm{ICH}(24 \mathrm{~h}$ ) group were shown. Scale bar $=10 \mu \mathrm{m}$. (f) The formation of necrosome was detected by immunoprecipitation (IP) by using anti-RIP1 antibody (rabbit immunoglobulin $\mathrm{G}(\mathrm{IgG})$ was also used as a negative control), and RIP3, MLKL and caspase-8 were detected by immunoblotting. The results suggested that increased interactions of RIP1 and RIP3, RIP1 and MLKL, and RIP1 and caspase-8 were observed in brain tissues at $24 \mathrm{~h}$ after ICH. Input, $5 \%$ of extract before IP. (g) Quantitative analysis of IP. ${ }^{* \star} P=0.0011$ versus Sham group; ${ }^{\& \&} P=0.0011$ versus Sham group; ${ }^{*} P=0.0152$ versus Sham group; all were unpaired $t$-test, $n=3$. Besides, results of IF (h) showed that expressions of these four proteins, which constituted necrosome, were increased significantly in brain tissues at $24 \mathrm{~h}$ after ICH than those in the Sham group. Scale bar $=100 \mu \mathrm{m}$. Related statistic of data was revealed in (i). ${ }^{* \star} P<0.0001$ versus Sham group; ${ }^{\& \&} P<0.0001$ versus Sham group; ${ }^{\# \#} P<0.0001$ versus Sham group; $\$ \$ P<0.0001$ versus Sham group; all were unpaired $t$ test, $n=6$. All data are expressed as means \pm S.E.M., mean value for the Sham group was normalized to 1.0
} 
d). These results indicated that knockdown of RIP1 can effectively block necroptotic pathway.

The results of BBB permeability also suggested that treatment with RIP1 siRNA in ICH rats can inhibit albumin extravasation and BBB permeability $(P=0.0493, n=3$; Figures $3 e$ and $\mathrm{f})$. The results of brain water content declared that, compared with the $\mathrm{ICH}$ group, the brain water content was significantly reduced in the RIP1 siRNA group $(P<0.0001$ in Ipsi-CX and $P=0.0209$ in Ipsi-BG, $n=6$; Figure $3 \mathrm{~g}$ ). The neurological score of the ICH+Si-RIP1 group was significantly lower than that of the ICH group $(P=0.0185, n=6$; Table 1$)$. The levels of TNF- $a$ in the CSF were detected by ELISA; the results confirmed that the level of TNF- $a$ was reduced by RIP1 siRNA treatment, indicating that downregulation of RIP1 could a

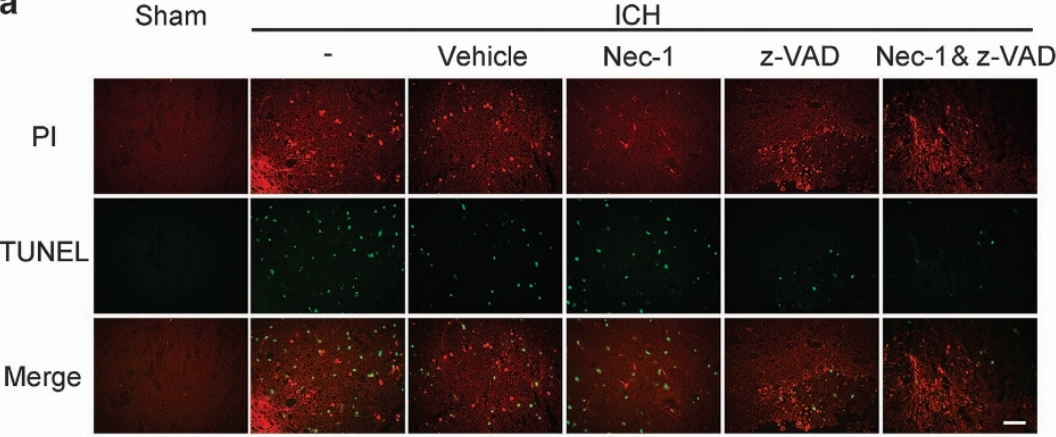

C

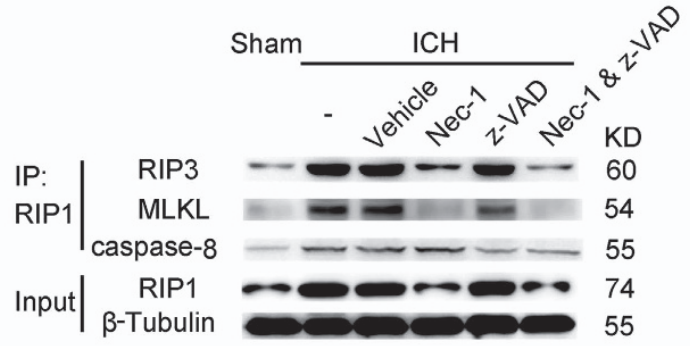

d

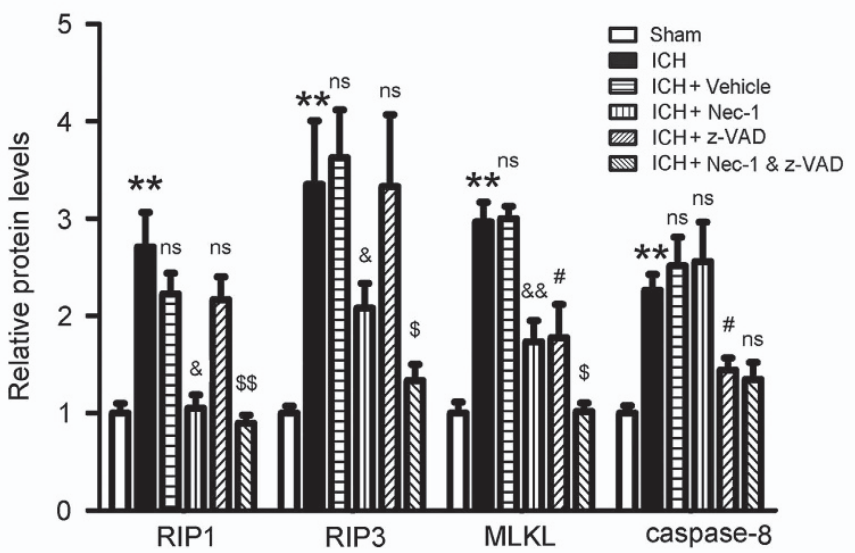

$\mathbf{g}$

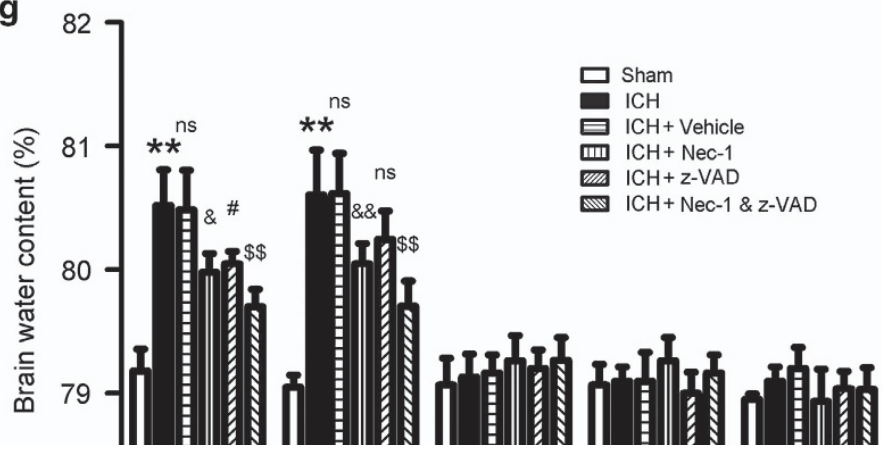

b

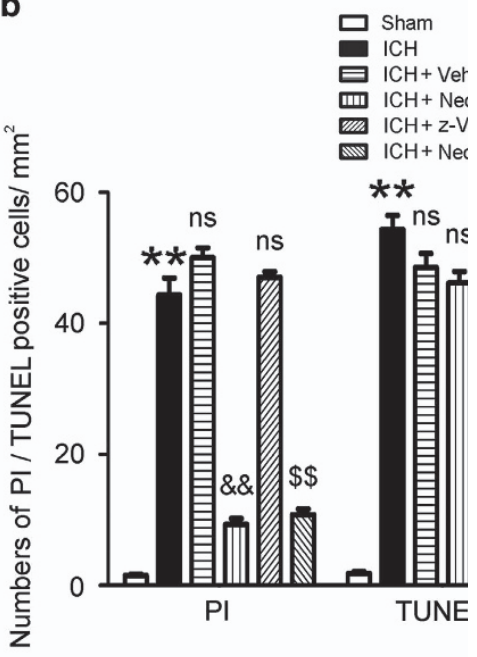

e

Sham

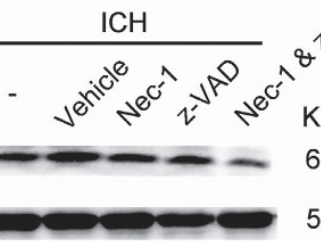

f
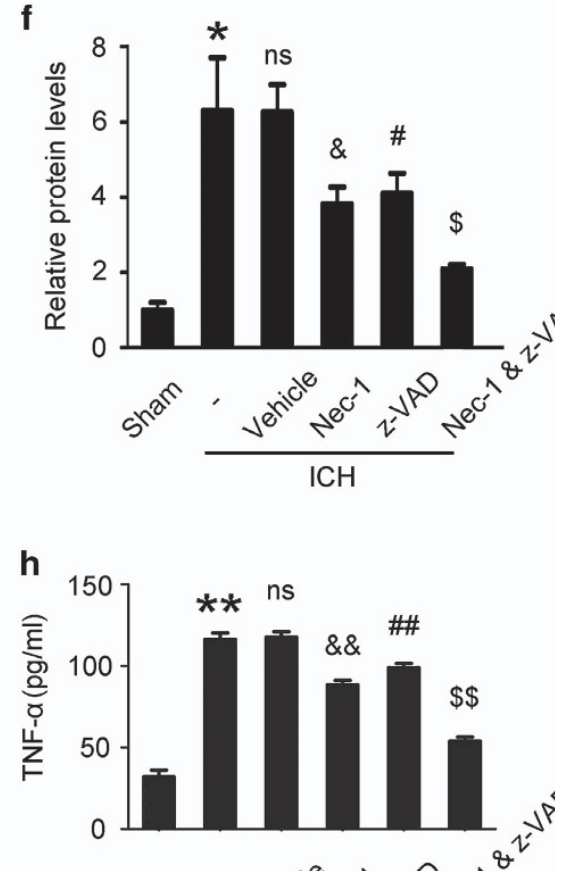
inhibit inflammation in brain tissues after $\mathrm{ICH}(P=0.0002$, $n=6$; Figure $3 h)$. These results further proved that necroptosis played an important role in brain injury after $\mathrm{ICH}$.

Conditioned medium from activated microglia can induce necroptosis of cultured neuron in vitro. To further explore the mechanism of necroptosis after $\mathrm{ICH}$, we used two in vitro models of $\mathrm{ICH}$ : one is oxygen hemoglobin $(\mathrm{OxyHb})$ to deal directly with the neurons, and the other is to prestimulate microglia with $\mathrm{OxyHb}$, collect the supernatant as conditioned medium and then treat neurons with the conditioned medium. After these treatments, neurons were digested by trypsin into cell suspension, stained with Annexin $\mathrm{V}$ and $\mathrm{PI}$ and then detected by flow cytometry. The results of flow cytometry

Table 1 Clinical behavior scores in each group $(n=6)$

\begin{tabular}{lc}
\hline Group & Score (mean \pm S.E.M.) \\
\hline Normal & $0.17 \pm 0.17$ \\
Sham & $0.33 \pm 0.21$ \\
ICH & $2.67 \pm 0.33^{\mathrm{a}}$ \\
ICH+vehicle & $3.17 \pm 0.31^{\mathrm{b}}$ \\
ICH+Nec-1 & $1.67 \pm 0.21^{\mathrm{c}}$ \\
ICH+Z-VAD & $1.83 \pm 0.17^{\mathrm{d}}$ \\
ICH+Nec-1 and z-VAD & $1.17 \pm 0.17^{\mathrm{e}}$ \\
ICH+Si-NC & $2.67 \pm 0.21^{\mathrm{f}}$ \\
ICH+Si-RIP1 & $1.67 \pm 0.21^{\mathrm{g}}$ \\
ICH+Ad-GFP & $3.00 \pm 0.37^{\mathrm{h}}$ \\
ICH+Ad-RIP1 & $4.33 \pm 0.21^{\mathrm{i}}$ \\
\hline
\end{tabular}

Abbreviations: Ad-GFP, adenovirus with GFP; Ad-RIP1, adenovirus with RIP1; $\mathrm{ICH}$, intracerebral hemorrhage; Nec-1, necrostatin-1; RIP1, receptor-interacting protein 1; Si-NC, Si-negative control.

${ }^{\text {a }} P=0.0043$ versus Sham group (Mann-Whitney test).

${ }^{\mathrm{b}} P=0.2959$ versus $\mathrm{ICH}$ group (unpaired $t$-test).

${ }^{c} P=0.0094$ versus $\mathrm{ICH}+$ vehicle group (Mann-Whitney test).

${ }^{\mathrm{d}} \mathrm{P}=0.0096$ versus $\mathrm{ICH}+$ vehicle group (Mann-Whitney test).

${ }^{\mathrm{e}} P=0.0341$ versus $\mathrm{ICH}+\mathrm{Z}-\mathrm{VAD}$ group (Mann-Whitney test).

${ }^{f} P=0.9290$ versus $\mathrm{ICH}$ group (Mann-Whitney test).

${ }^{\mathrm{g}} P=0.0185$ versus $\mathrm{ICH}+\mathrm{Si}-\mathrm{NC}$ group (Mann-Whitney test).

${ }^{\mathrm{h}} P=0.5155$ versus $\mathrm{ICH}$ group (unpaired $t$-test).

i $P=0.0205$ versus $\mathrm{ICH}+$ Ad-GFP group (Mann-Whitney test). showed that, compared with control group, there was a higher apoptotic ratio $(27.8 \%)$ in $\mathrm{OxyHb}$ directly stimulated neuron group, whereas there was a higher necroptotic ratio in conditioned medium treatment group ( $31.5 \%)$. In addition, Nec-1 treatment, but not z-VAD, significantly reduced the conditioned medium-induced neuron necroptosis (data not shown). However, the TNF- $a$ inhibitor pretreatment significantly reduced the percentage of necroptotic neurons ( 13.5\%; all $P<0.01, n=3$; Figures $4 \mathrm{a}$ and $\mathrm{b}$ ).

The results of IP also demonstrated that, compared with the control, interactions of RIP1 and RIP3, RIP1 and MLKL, and RIP1 and caspase- 8 were significantly increased by treatment with conditioned medium. However, interactions of RIP1 and RIP3, RIP1 and MLKL, and RIP1 and caspase-8 were remarkably inhibited by TNF- $a$ inhibitor pretreatment than that in the conditioned medium group (all $P<0.01, n=3$; Figures $4 \mathrm{c}$ and $\mathrm{d}$ ). To further clarify the interaction among RIP1-RIP3-MLKL-caspase-8, immunofluorescence staining of RIP1/MLKL/caspase-8 and RIP3/MLKL/caspase-8 was also performed on primary neurons (Figure $4 \mathrm{e}$ ). Consistent with the results of IP, there were increased colocalizations of RIP1-MLKL-caspase-8 and RIP3-MLKL-caspase in conditioned medium-treated neurons, which were significantly inhibited by TNF- $a$ inhibitor. PI and Hoechst staining also showed that treatment with conditioned medium increased the ratio of necroptosis in neurons, but this can be inhibited by TNF- $a$ inhibitor pretreatment (both $P<0.0001, n=6$; Figures $4 \mathrm{f}$ and $\mathrm{g}$ ). These results suggested that TNF- $a$ in conditioned medium may be an important factor of inducing necroptosis in neurons.
Phosphorylation of RIP1 has an essential role in activa- tion of necroptosis in neuron in vitro. Phosphorylation of RIP1 is an essential element of activation of necroptosis. ${ }^{15}$ We detected the phosphorylation of RIP1 by IP using anti- RIP1 antibody followed by immunoblotting for anti- phosphorylation serine ( $p$-Ser) antibody. The results of IP suggested that the phosphorylation (serine site) of RIP1 was

\begin{abstract}
Figure 2 Different effects of Nec-1 and z-VAD on brain injury after ICH. Nec-1 (the specific inhibitor of necroptosis) and z-VAD (a caspase inhibitor) were used to explore whether necroptosis contributes to brain injury after ICH. (a) The necroptosis in cells were detected by PI staining and apoptosis by terminal deoxynucleotidyl transferase dUTP nick-end labeling (TUNEL) staining. Nec-1 reduced the PI+ cells, whereas it had no significant effects on TUNEL+ cells (apoptosis), and z-VAD only downregulated the TUNEL+ cells. Additionally, combination of Nec-1 and z-VAD obviously both reduced the PI+ cells and TUNEL+cells. Scale bar $=100 \mu \mathrm{m}$. (b) Corresponding bar graph revealed relative levels of $\mathrm{PI} / \mathrm{TUNEL}+$ cells. ${ }^{* \star} P<0.0001$ (both in $\mathrm{PI}$ and TUNEL) versus Sham group; NS, not significant difference $(P=0.0909$ in $\mathrm{PI}$ and $P=0.0857$ in TUNEL) versus ICH group; ${ }^{\& \&} P<0.0001$ in $\mathrm{PI}$ and NS $(P=0.4233)$ in TUNEL versus vehicle group; NS $(P=0.4233)$ in $\mathrm{PI}$ and ${ }^{\# \#} P<0.0001$ in TUNEL versus vehicle group; $\$ P<0.0001$ in $\mathrm{PI}$ and NS $(P=0.0663)$ in TUNEL versus z-VAD group; all were unpaired $t$-test, $n=6$. (c) IP showed that Nec-1 reduced interactions of RIP1 and RIP3, RIP1 and MLKL, and RIP1 and caspase-8, indicating that the formation of necrosome was inhibited. Input, $5 \%$ of extract before IP. (d) Corresponding bar graph revealed relative levels of association. ${ }^{\star \star} P=0.0097$ in RIP1, ${ }^{\star \star} P=0.0099$ in RIP3, ${ }^{\star \star} P=0.0010$ in MLKL and ${ }^{\star \star} P=0.0022$ in caspase-8 versus Sham group; NS, not significant difference $(P=0.3077$ in RIP1, $P=0.7942$ in RIP3, $P=0.9047$ in MLKL and $P=0.4930$ in caspase-8) versus ICH group; ${ }^{8} P=0.0102$ in $R I P 1,{ }^{\&} P=0.0493$ in $R I P 3, \& \& P=0.0074$ in $M L K L$ and NS $(P=0.9349)$ in caspase-8 versus vehicle group; NS $(P=0.8658)$ in RIP1, NS $(P=0.6857)$ in RIP $3,{ }^{\#} P=0.0205$ in MLKL and ${ }^{\#} P=0.0281$ in caspase-8 versus vehicle group; ${ }^{\$}$ ${ }^{\$} P=0.0065$ in RIP1, ${ }^{\$} P=0.0499$ in RIP3,${ }^{\$} P=0.0403$ in MLKL and NS $(P=0.6746)$ in caspase-8 versus z-VAD group; all were unpaired $t$-test, $n=3$. (e) Expression of albumin, which is regarded as the index of BBB injury, was increased after ICH, whereas it could be significantly decreased when treated with Nec-1 and/or z-VAD. (f) Corresponding bar graph revealed relative levels of albumin. ${ }^{\star} P=0.0200$ versus Sham group; NS, not significant difference $(P=0.9779)$ versus ICH group; ${ }^{\star} P=0.0461$ versus vehicle group; ${ }^{\#} P=0.0496$ versus vehicle group; ${ }^{\$} P=0.0126$ versus $z$-VAD group; all were unpaired $t$-test, $n=6$. (g) Compared with ICH group, the brain water content was partially attenuated in Nec-1 and/or z-VAD group, ${ }^{\star \star} P<0.0001$ (both in Ipsi-CX and Ipsi-BG) versus Sham group; NS, not significant difference $(P=0.9022$ in Ipsi-CX and $P=0.9660$ in Ipsi-BG) versus ICH group; ${ }^{\&} P=0.0109$ in Ipsi-CX and ${ }^{\& \&} P=0.0077$ in Ipsi-BG versus vehicle group; ${ }^{\#} P=0.0162$ in Ipsi-CX and NS $(P=0.0727)$ in Ipsi-BG versus vehicle group; ${ }^{\$}$ $\$ P=0.0015$ in Ipsi-CX and ${ }^{\$} P=0.0039$ in Ipsi-BG versus z-VAD group; all were unpaired t-test, $n=6$. (h) The levels of TNF- $\alpha$ in the CSF were measured by ELISA and reduced by Nec-1 treatment. ${ }^{* \star} P<0.0001$ versus Sham group, NS, not significant difference $(P=0.7897)$ versus ICH group; ${ }^{\&} P<0.0001$ versus vehicle group, ${ }^{\# \#} P=0.0008$ versus vehicle group, ${ }^{\$} P<0.0001$ versus $z-V A D$ group; all were unpaired $t$-test, $n=6$. All data are expressed as means $\pm S$.E.M. and mean value for Sham group was normalized to 1.0 .
\end{abstract}


obviously increased in the conditioned medium treatment group and could be inhibited by TNF- $a$ inhibitor pretreatment (both $P<0.01, n=3$; Figures $4 \mathrm{c}$ and d). To further explore the molecular mechanism of RIP1 in necroptosis after $\mathrm{ICH}$, we used overexpression with a mutation of phosphorylation site (S166A) of RIP1. After transfection and being cultured for another $24 \mathrm{~h}$, neurons were digested by trypsin into cell suspension, stained with Annexin $\mathrm{V}$ and $\mathrm{PI}$ and then detected a

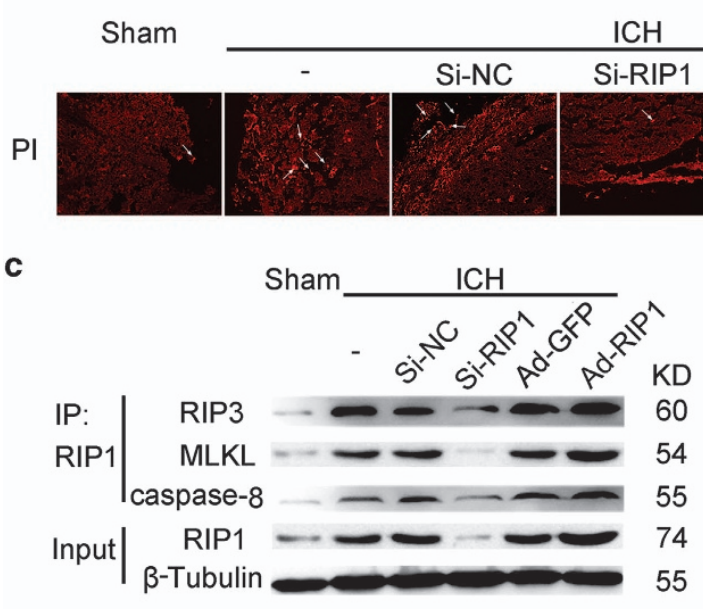

d

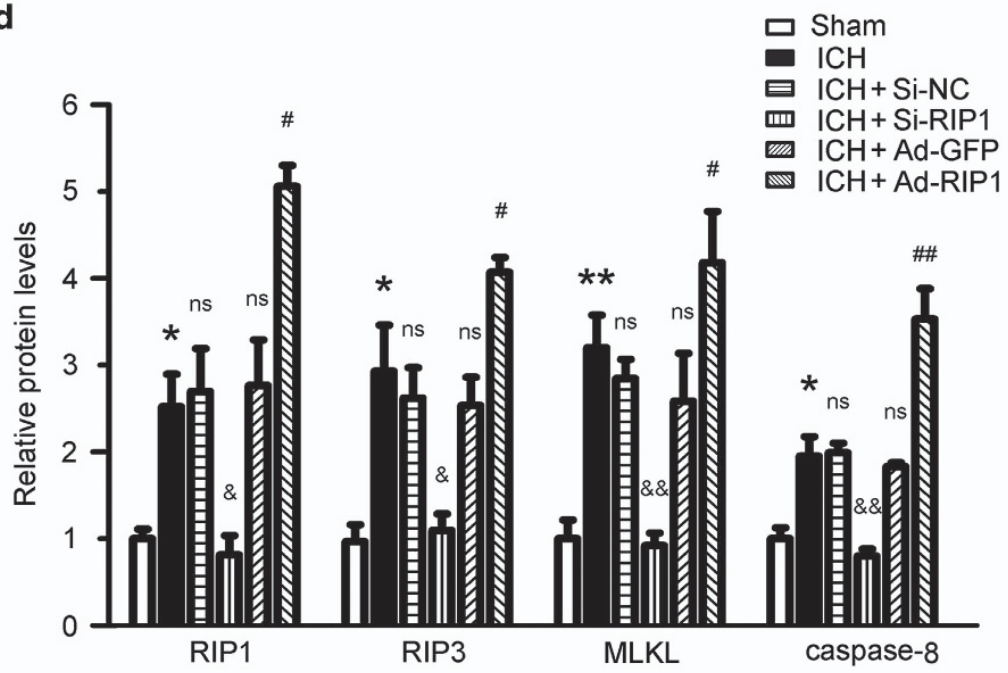

9

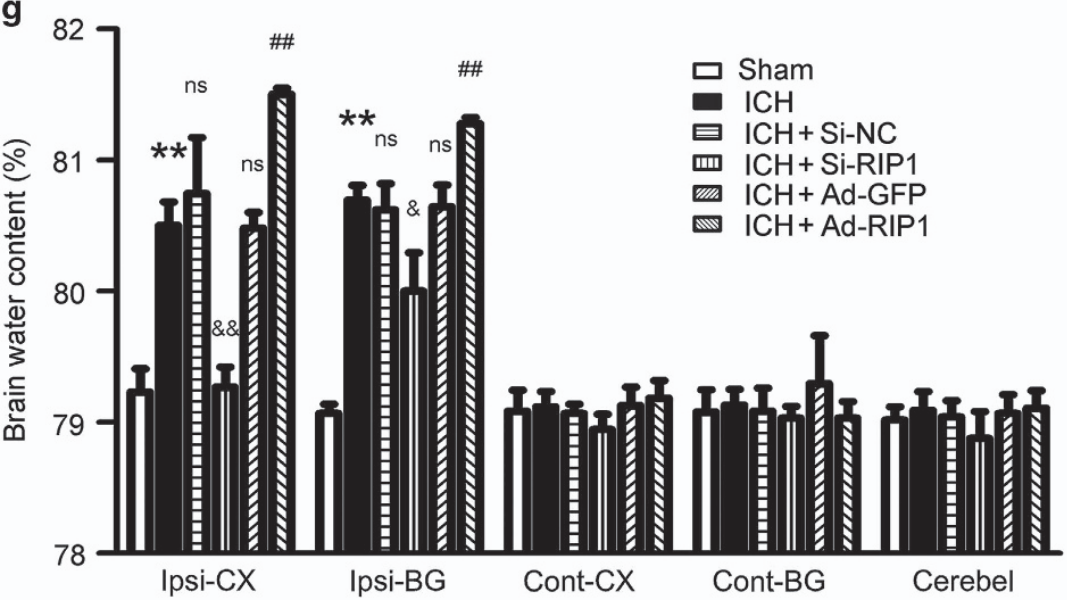

b

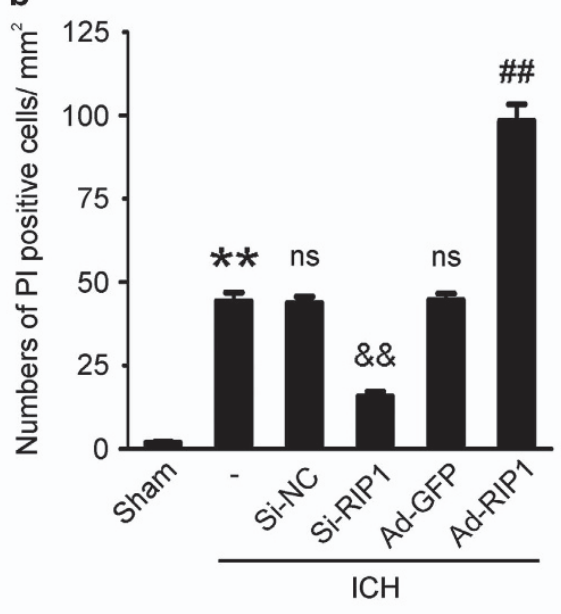

e
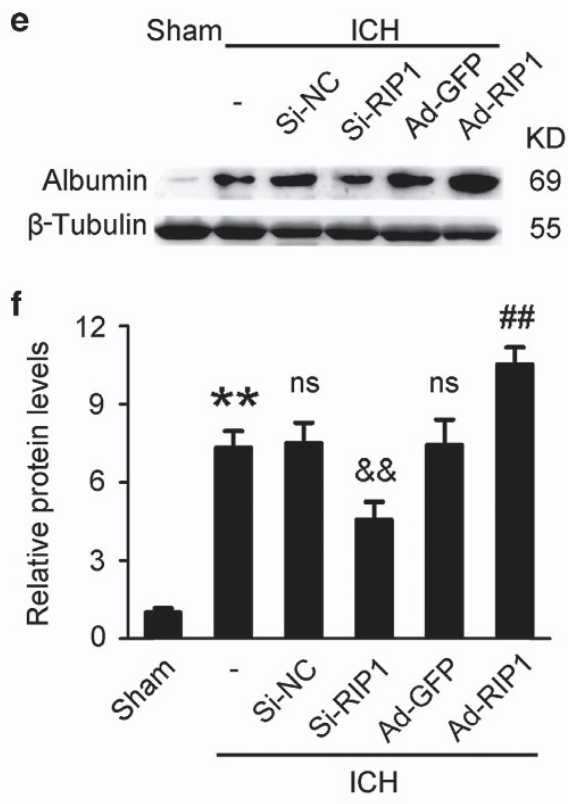

h \#\#

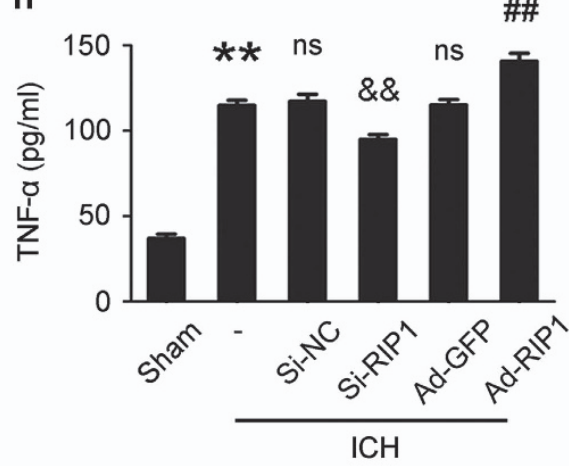


by flow cytometry. The results of flow cytometry revealed that upregulating the expression of RIP1 could increase the percentage of necroptosis ( $66.7 \%)$ in neurons, but overexpression of mutation of phosphorylation site (S166A) of RIP1 has no obvious effect in inducing necroptosis of neurons $(\sim 34.2 \%)$, and Nec-1 treatment can also inhibit the necroptosis induced by RIP1 overexpression $(\sim 21.7 \%$, all $P<0.01, n=3$; Figures $5 \mathrm{a}$ and $\mathrm{b}$ ).

The results of IP also demonstrated that, compared with the control, interactions of RIP1 and RIP3, RIP1 and MLKL, and RIP1 and caspase-8 were significantly increased by overexpression of RIP1. However, interactions of RIP1 and RIP3, RIP1 and MLKL, and RIP1 and caspase-8 were remarkably inhibited by mutation of phosphorylation site (S166A) of RIP1 than those in the overexpression of wild type of the RIP1 group (all $P<0.01, n=3$; Figures $5 \mathrm{c}$ and $\mathrm{d}$ ). $\mathrm{Pl}$ and Hoechst staining also showed that overexpression of wild-type RIP1 increases the ratio of necroptosis in neurons, but this effect can be inhibited by mutation of the phosphorylation site (S166A) of RIP1 (all $P<0.0001, n=6$; Figures $5 e$ and f). These results all suggested that phosphorylation in the 166th site of RIP1 may be an important element of inducing necroptosis in neurons.

\section{Discussion}

Cell death is the important reason leading to brain injury after $\mathrm{ICH}$. Almost previous studies believed that the form of cell death is apoptosis in brain tissues after $\mathrm{ICH}$; few researches put attention to the role of necrosis. Initiation factors of apoptosis include downregulation of blood flow and energy metabolism around the hematoma; a variety of enzymes that are activated in the blood after $\mathrm{ICH}$ activate the apoptosis signal, and mechanical damage of hematoma directly causes apoptosis. ${ }^{5,16,17}$ Apoptosis theory can partly explain the mechanism of cell death in brain tissues after $\mathrm{ICH}$. However, recent study found that the dead brain cells release a series of proteins from the cytoplasm after $\mathrm{ICH}$; these proteins were known as danger-associated molecular patterns. The most typical representative is the high-mobility group protein 1 , which can stimulate the inflammatory response that aggravates secondary brain injury after $\mathrm{ICH} .{ }^{18}$ These results pose challenge to apoptosis theory in $\mathrm{ICH}$; scholars widely recognized that cell apoptotic process and released apoptotic bodies do not cause inflammation. ${ }^{15}$ Thus, presumably, another form of cell death in addition to apoptosis might exist in brain tissues and can stimulate inflammatory response after $\mathrm{ICH}$. On the other hand, microglia were rapidly activated after $\mathrm{ICH}$ and released substantial inflammatory factors (such as TNF-a); previous studies have confirmed that TNF- $a$ can not only induce apoptosis but also necroptosis; so whether necroptosis exists in brain tissues after $\mathrm{ICH}$ becomes a question.

Our present study showed that conditioned medium, especially the key composition TNF- $\alpha$, from activated microglia induced necrosis of neurons after $\mathrm{ICH}$ for the first time. We found that necroptosis existed in brain tissues after $\mathrm{ICH}$, and it had an important role in neuronal dysfunction, brain edema and BBB permeability after ICH. RIP1 inhibitor Nec-1 can remarkably attenuate neurological dysfunction, brain edema and BBB injury, and combinational use with apoptosis inhibitor z-VAD has a better effect, suggesting that necroptotic pathway and RIP1 may become targets for the treatment of brain injury after $\mathrm{ICH}$. We also observed that treatment of $\mathrm{ICH}$ rats with Nec-1 does not affect the apoptosis of brain cells, and the use of $\mathrm{z}$-VAD treatment of $\mathrm{ICH}$ rats also does not affect the necroptosis in brain tissues; these results indicated that apoptosis and necroptosis were coexisting and were relatively independent after $\mathrm{ICH}$. Nec-1 and z-VAD could inhibit apoptosis and necroptosis, respectively, whereas combinational treatment of $\mathrm{Nec}-1$ and $\mathrm{z}-\mathrm{VAD}$ was more effective in treating $\mathrm{ICH}$ than each inhibitor alone. In addition, there may be a crosstalk between apoptosis and necroptosis in the progression of $\mathrm{ICH}$, which needs further investigation. In addition, while our research was in progress, Su et al. ${ }^{19}$ reported that Nec-1 ameliorated brain injury after $\mathrm{ICH}$ in a collagenase-induced $\mathrm{ICH}$ model in mice. ${ }^{19} \mathrm{~A}$ recent report also showed that $\mathrm{Nec}-1$ reduced neurovascular injury after $\mathrm{ICH}$ in a collagenase-induced $\mathrm{ICH}$ model in mice. ${ }^{20}$ As our present study showed that, TNF- $a$, an important inflammatory

Figure 3 Knockdown of RIP1 reduced necroptosis in brain tissues after ICH. Knockdown and overexpression of RIP1 were used to study the role of RIP1-mediated necroptosis after ICH in rats. (a) $\mathrm{PI}+$ cells decreased in the Si-RIP1 group (knockdown), whereas they increased in the Ad-RIP1 group (overexpression). Arrows point to PI+ cells. Scale bar $=200 \mu \mathrm{m}$. (b) Related with (a), it revealed relative levels of PI+ cells. ${ }^{* \star} P<0.0001$ versus Sham group; NS, not significant difference $(P=0.8453)$ versus ICH group; ${ }^{\& \&} P<0.0001$ versus Si-NC group; NS, not significant difference $(P=0.9211)$ versus ICH group; ${ }^{\#} P<0.0001$ versus Ad-GFP group; all were unpaired t-test, $n=6$. (c) IP demonstrated that interactions of RIP1 and RIP3, RIP1 and MLKL, and RIP1 and caspase-8 were significantly inhibited in the RIP1-knockdown group, whereas they increased in the overexpression group. Input, $5 \%$ of extract before IP. Quantitative analysis of IP was shown in (d), ${ }^{*} P=0.0177$ in RIP1, ${ }^{*} P=0.0261$ in $\mathrm{RIP} 3,{ }^{* \star} P=0.0072$ in MLKL and ${ }^{*} P=0.0209$ in caspase-8 versus Sham group; NS, not significant difference ( $P=0.7939$ in RIP1, $P=0.6515$ in RIP3, $P=0.4640$ in MLKL and $P=0.8812$ in caspase-8) versus ICH group; ${ }^{\&} P=0.0254$ in RIP1, ${ }^{\&} P=0.0184$ in RIP3, ${ }^{\text {\&\& }} P=0.0019$ in MLKL and ${ }^{\& \&} P=0.0010$ in caspase-8 versus Si-NC group; NS $(P=0.7254)$ in RIP1, NS $(P=0.5590)$ in RIP3, NS $(P=0.3154)$ in MLKL and NS $(P=0.6284)$ in caspase-8 versus ICH group; ${ }^{\#} P=0.0164$ in RIP1, ${ }^{\#} P=0.0138$ in RIP3, ${ }^{\#} P=0.0367$ in MLKL and ${ }^{\# \#} P=0.0083$ in caspase-8 versus Ad-GFP group; all were unpaired $t$-test, $n=3$. (e) Expression of albumin was elevated in the Ad-RIP1 group, whereas it was opposite in the Si-RIP1 group. (f) Bar graph related to (e). ${ }^{*} P=0.0007$ versus Sham group; NS, not significant difference $(P=0.8780)$ versus ICH group; ${ }^{\&} P=0.0493$ versus Si-NC group; NS, not significant difference $(P=0.9361)$ versus ICH group; ${ }^{\#} P=0.0470$ versus Ad-GFP group; all were unpaired $t$-test, $n=3$. (g) Brain water content decreased in the Si-RIP1 group, whereas it was opposite in the Ad-RIP1 group, ${ }^{\star *} P<0.0001$ (both in Ipsi-CX and Ipsi-BG) versus Sham group; NS, not significant difference $(P=0.4041$ in Ipsi-CX and $P=0.5003$ in IpsiBG) versus ICH group; ${ }^{\& \&} P<0.0001$ in Ipsi-CX and ${ }^{\&} P=0.0209$ in Ipsi-BG versus $S \mathrm{i}-\mathrm{NC}$ group; NS, not significant difference $(P=0.5276$ in Ipsi-CX and $P=0.6015$ in Ipsi-BG) versus ICH group; ${ }^{\# \#} P<0.0001$ (both in Ipsi-CX and Ipsi-BG) versus Ad-GFP group; all were unpaired t-test, $n=6$. (h) The levels of TNF- $\alpha$ in the CSF were reduced in RIP1knockdown group, whereas the effect was diametric in the RIP1 overexpression group. ${ }^{* *} P<0.0001$ versus Sham group; NS, not significant difference $(P=0.5570)$ versus ICH group; ${ }^{\text {\& }} P=0.0002$ versus Si-NC group; NS, not significant difference $(P=0.9151)$ versus ICH group; ${ }^{\#} P<0.0001$ versus Ad-GFP group; all were unpaired t-test, $n=6$. All data are expressed as means \pm S.E.M., and mean values for Sham group were normalized to 1.0. Ad-GFP, adenovirus with GFP; Ad- RIP1, adenovirus with RIP1; Si-NC, Si-negative control. 
cytokine, may be a key factor in neurons' necrosis after $\mathrm{ICH}$, the side effects of collagen on inflammatory response should not be ignored in necrosis-related studies. In this study, we researched neurons' necrosis in autologous blood injection $\mathrm{ICH}$ model for the first time.
In many previous studies, stimulation of neurons with $\mathrm{OxyHb}$ was an in vitro model of $\mathrm{ICH}^{21}$ However, the inflammation is an important event in second brain injury after $\mathrm{ICH}$; activated microglia released an amount of inflammatory cytokines such as TNF- $\alpha$ and IL-1 $\beta$, which promote brain injury a
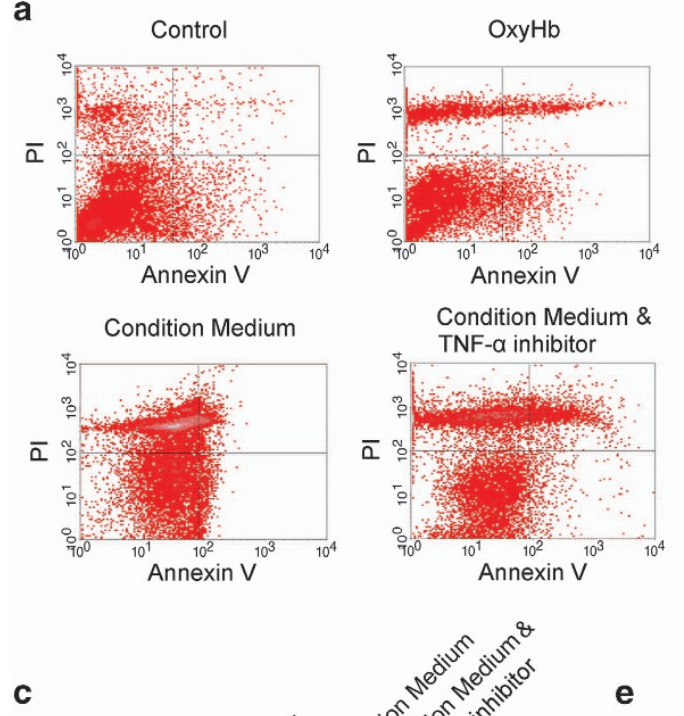

Condition Medium \&

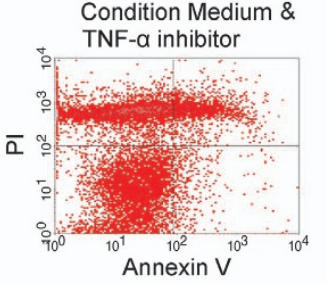

e b

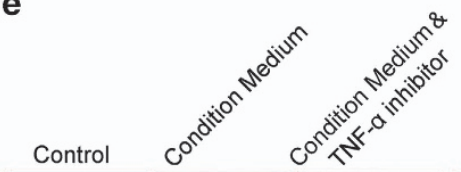

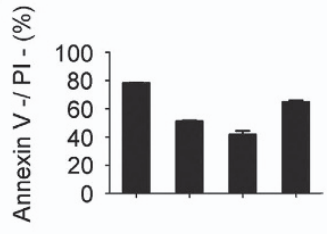

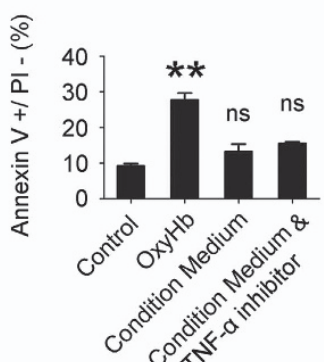

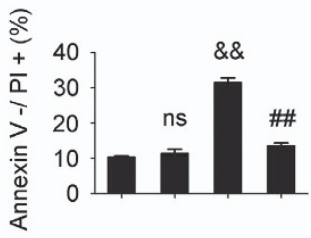

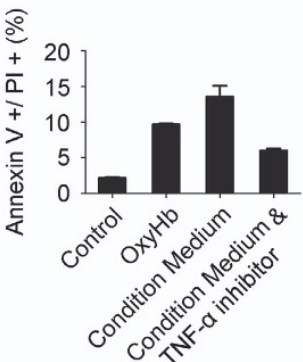

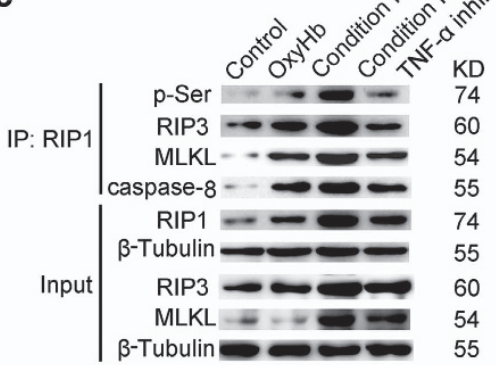
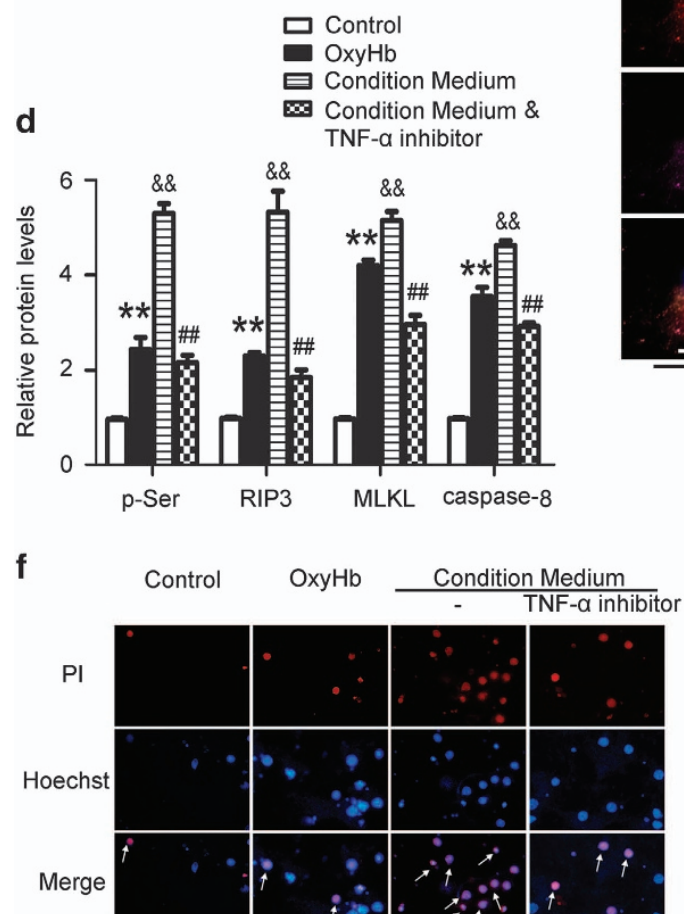

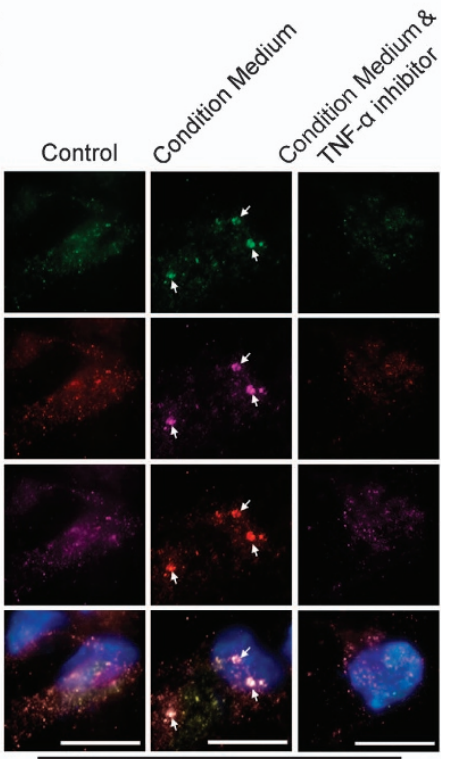

RIP3/MLKL/caspase-8/DAPI

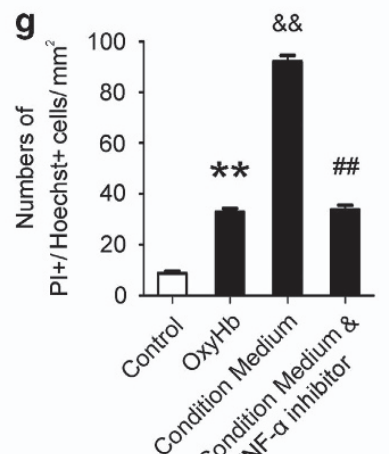


after $\mathrm{ICH}^{22}$ Thus, in this study, we used OxyHb-stimulated microglia and collected the supernatant as conditioned medium, and then used conditioned medium to treat the neurons, as an $\mathrm{ICH}$ model in vitro. This model highlights the stimulation of inflammatory factor on neurons, and results of ELISA also suggested that the level of TNF- $a$ was significantly increased in conditioned medium compared with the medium of nontreated microglia (data not shown). We found that necroptotic rate of neurons in this model was significantly higher than that in the $\mathrm{OxyHb}$-treated neurons, suggesting that inflammatory factors released by activated microglia are a key factor leading to cell necroptosis in brain tissues. A previous study reported that, in a murine model of $\mathrm{ICH}$, postinjury treatment with the TNF- $a$ antibody resulted in less neuroinflammation and reduction in functional deficit; ${ }^{23}$ combining with our results, we can conclude that ameliorating brain injury after ICH by blocking TNF- $\alpha$ is partially because of reducing the necroptosis in brain tissues. Our results also suggested that multiple stimuli factors coexisted in brain tissues after $\mathrm{ICH}$, and led to cell death by different pathways; the dominant stimulus in local microenvironment might be the main reason leading to brain cell death. At the same time, our results demonstrated that use of multiple in vitro models may be more appropriate in the study of brain injury following $\mathrm{ICH}$.

Unlike apoptosis, the release of cell contents will cause inflammation after the cell necroptosis. Interestingly, an important initial factor of necroptosis is stimulation of inflammatory factor (such as TNF-a), and necroptosis also can further promote the inflammation, these suggested that possibly have a positive feedback relationship between necroptosis and inflammation in brain injury after $\mathrm{ICH}$. The effect of this positive feedback relationship in the pathophysiological processes after ICH needs to be further confirmed. There are also some deficiencies in this study; although the role of necroptosis in the brain injury following $\mathrm{ICH}$ was explored, the molecular mechanism is still uncertain. On the other hand, there are many kinds of inflammatory cytokines released by activated microglia, such as IL-1 $\beta$, IL- 6 and IL-18; whether these inflammatory factors also can cause cell necroptosis in brain tissues after $\mathrm{ICH}$ is not clear.

In summary, our study confirmed that the RIP1-mediated necroptosis exists in brain tissues, and it has an important role in brain injury following $\mathrm{ICH}$; on the other hand, we used the in vitro model of $\mathrm{ICH}$ suggesting that the release of TNF-a from activated microglia might be an important factor inducing necroptosis in ICH (Figure 6). These findings further revealed the causes of cell death and the relationship between cell death and inflammation in brain injury after $\mathrm{ICH}$ and provide a potential therapeutic target for secondary brain injury after $\mathrm{ICH}$.

\section{Materials and Methods}

Animals. Adult male Sprague-Dawley (SD) rats, weighing $\sim 300 \mathrm{~g}$, were provided by the Animal Center of Chinese Academy of Sciences (Shanghai, China). Experimental protocols were approved by the Animal Care and Use Committee of Soochow University, and were implemented with reference to the National Institutes of Health guidelines. The animals were freely fed and housed in a quiet environment (indoor temperature of $\sim 18-22{ }^{\circ} \mathrm{C}$ ). Additionally, we strived as much as possible to minimize the number of animals that were used and reduce their suffering. Besides, primary neuronal and microglial cultures (in vitro) were prepared using 16-18-day-old pregnant SD rats.

Experimental design. In experiment 1, 54 rats (70 rats were used, 54 rats survived after the surgery) were randomly assigned to nine groups of six rats each; the normal group, the Sham group and seven experimental groups were arranged by time $-3,6,12,24,48,72 \mathrm{~h}$ and 7 days after $\mathrm{ICH}$. Arriving at separate time points after SAH, all rats were killed and cerebral tissue samples were collected for analysis (Figure 7a). In experiment 2, 60 rats (75 rats were used, 60 rats survived after the surgery) were randomly divided into 10 groups - the Sham group, the $\mathrm{ICH}$ group, the ICH+vehicle group, the $\mathrm{ICH}+\mathrm{Nec}-1$ group, the $\mathrm{ICH}+\mathrm{z}-\mathrm{VAD}$ group, the $\mathrm{ICH}$ $+\mathrm{Nec}-1+z-V A D$ group, the $\mathrm{ICH}+\mathrm{Si}-$ negative control group, the ICH+SiRNA-RIP1 group, the ICH+Ad-GFP group and the ICH+Ad-RIP1 group. At $24 \mathrm{~h}$ after $\mathrm{ICH}$, all rats were examined for behavioral impairment, and then brain samples were collected (Figure 7b). In experiment 3, primary cultured neurons were used and partitioned into six groups - the control group, the OxyHb group, the conditioned medium group, the conditioned medium+TNF- $\alpha$ inhibitor group, the conditioned medium+Ad-RIP1 group and the conditioned medium+Ad-RIP1-S166A group (Figure 7c). Detailed information about each group was shown in special procedures as below.

Establishment of ICH model. ICH model in vivo was established by injection of autologous blood. ${ }^{24}$ After anesthesia with intraperitoneal injection of $4 \%$

\footnotetext{
Figure 4 Conditioned medium-induced necroptosis in neurons in vitro. We used two in vitro ICH models to further explore the role of inflammatory factors, such as TNF- $\alpha$, in inducing necroptosis: one is neurons treated with $\mathrm{OxyHb}$ directly, and the other is using supernatant of culture of microglia (stimulated with $\mathrm{OxyHb}$ in advance) as neurons' conditioned medium, and treated with or without inhibitor of TNF- $\alpha$. (a) The necroptosis and apoptosis of neurons in vitro were detected by PI and Annexin V double staining and flow cytometry analysis, respectively. PI - /Annexin V - represented survival neurons, Pl+/Annexin V - represented necroptotic neurons, $\mathrm{PI}-/$ Annexin $\mathrm{V}+$ represented apoptotic neurons, and $\mathrm{Pl}+/$ Annexin $\mathrm{V}+$ represented a mixed damage of neurons. The results of flow cytometry indicated a higher ratio ( 27.8\%) of apoptosis and a lower ratio $(\sim 11.4 \%)$ of necroptosis when neurons were stimulated with OxyHb. However, in conditioned medium treatment group, it was a higher percentage of necroptosis $(\sim 31.5 \%)$, whereas the ratio could be significantly reduced when treated with TNF- $\alpha$ inhibitor $(\sim 13.5 \%)$. (b) Related bar graph showed four different conditions of neurons in various groups; NS, not significant difference $(P=0.4642)$ in $\mathrm{Pl}+/$ Annexin $\mathrm{V}-$ cells and ${ }^{*} P=0.0007$ in $\mathrm{PI}-/$ Annexin $\mathrm{V}+$ cells versus Control group; ${ }^{\& \&} P=0.0001$ in $\mathrm{PI}+/ \mathrm{Annexin} \mathrm{V}-$ cells and NS, not significant difference $(P=0.1498)$ in $\mathrm{PI}-/$ Annexin $\mathrm{V}+$ cells versus Control group; ${ }^{\# \# ~} P=0.0004$ in $\mathrm{PI}+/$ Annexin $\mathrm{V}-$ cells and NS, not significant difference $(P=0.3401)$ in $\mathrm{PI}$ - /Annexin V+ cells versus Conditioned medium group; all were unpaired $t$-test, $n=3$. (c) IP revealed that when treated with conditioned medium, interactions of RIP1 and RIP3, RIP1 and MLKL, and RIP1 and caspase-8 were remarkably increased. And these results were attenuated when pretreated with TNF- $\alpha$ inhibitor. (d) Consistent data

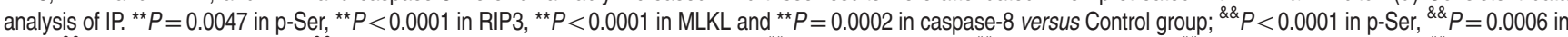
RIP3, ${ }^{\text {\& }} P<0.0001$ in MLKL and ${ }^{\text {\& }} P<0.0001$ in caspase-8 versus Control group; ${ }^{\# \#} P=0.0003$ in $p$-Ser, ${ }^{\# \#} P=0.0018$ in RIP3, ${ }^{\# \#} P=0.0012$ in MLKL and ${ }^{\# \#} P=0.0002$ in caspase-8 versus conditioned medium group. The mean values for the control group were normalized to 1.0, all were unpaired $t$-test, $n=3$. (e) Immunofluorescence analysis was performed with antibody for RIP1/RIP3 (green), MLKL (red) and caspase-8 (purple) in cultured primary neurons under indicated treatment. Nuclei were fluorescently labeled with DAPI (blue). Representative images were shown. Arrows indicated the colocalization of RIP1-MLKL-caspase-8 and RIP3-MLKL-caspase. Scale bar $=20 \mu \mathrm{m}$. (f) PI and Hoechst double staining was also used in detection of necroptosis. The results showed that neurons in conditioned medium had higher ratio of necroptosis (as arrows point to, $\mathrm{Pl}+/ \mathrm{Hoechst}$

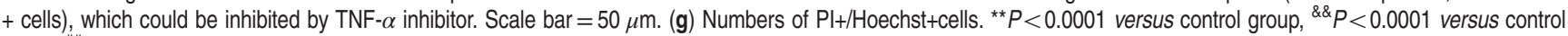
group, ${ }^{\# \#} P<0.0001$ versus conditioned medium group; all were unpaired $t$-test, $n=6$. All data are expressed as means \pm S.E.M.
} 
chloral hydrate at a dosage of $1 \mathrm{ml} / 100 \mathrm{~g}, 100 \mu \mathrm{l}$ of autologous blood was collected from the heart, and then the rats were fixed in the stereotaxic frame (Zhenghua Biological Equipment Co. Ltd, Anhui, China). The scalp was exposed and made drilling a hole corresponding to right basal ganglia $(0.2 \mathrm{~mm}$ anterior to the intersection between the coronal suture and sagittal midline and $3.5 \mathrm{~mm}$ to the right of the sagittal suture). A microsyringe was affixed to the stereotactic frame and a needle was slowly inserted (5.5 mm in depth), and then $100 \mu$ l of autologous blood was slowly injected $(20 \mu / / \mathrm{min})$. Before slowly withdrawing the needle, it was kept in place for another $5 \mathrm{~min}$. Rats in the Sham group were intracerebrally injected with $100 \mu$ l physiological saline solution. The bone hole was sealed with bone wax, and

a

Condition Medium
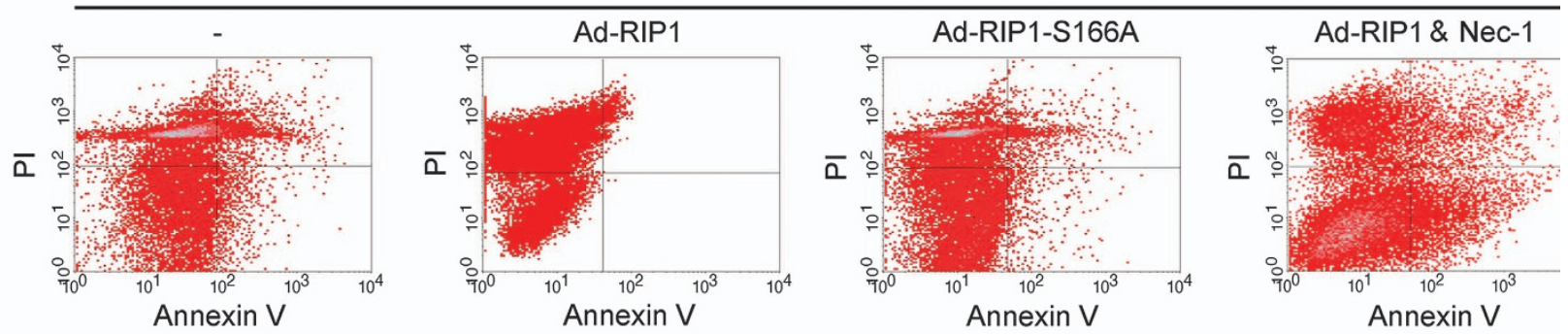

b
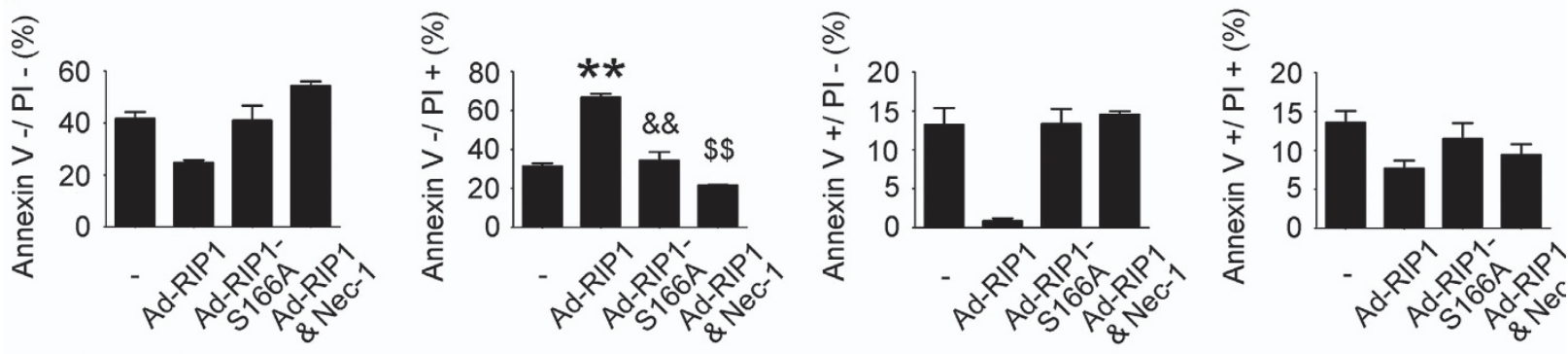

Condition Medium

C

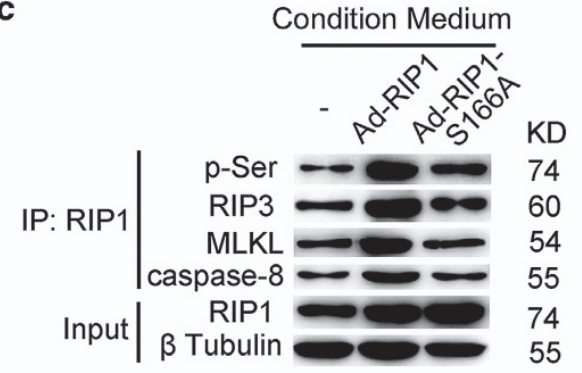

e

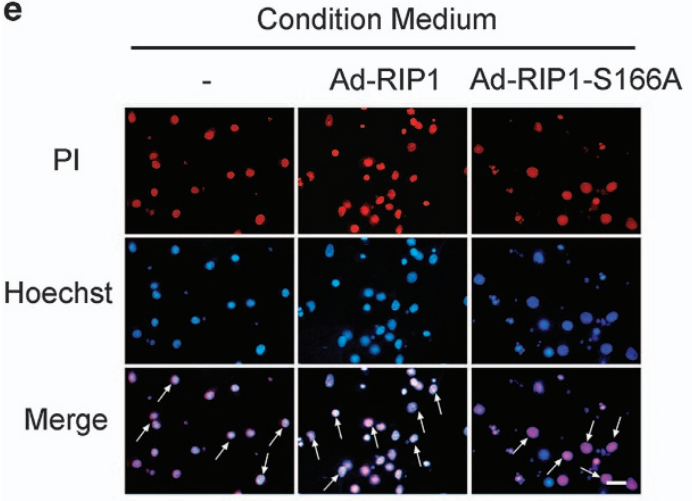

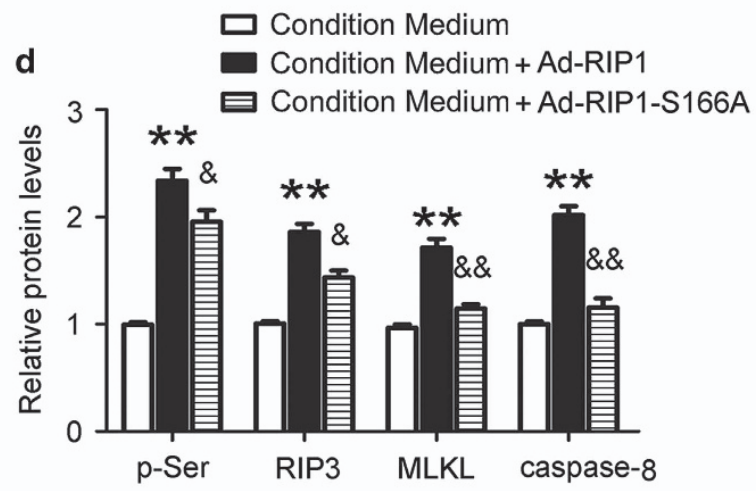

f

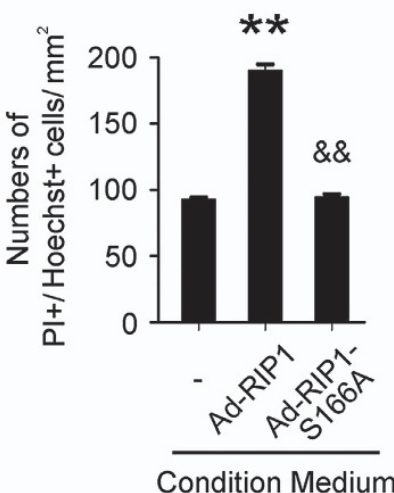


skin incision was disinfected and sutured. During the establishment of the model, rats' vital signs were monitored and maintained in normal level.

Primary neuron- and microglia-enriched cultures. Neuron-enriched cultures were prepared from brains of fetal rats (from 16-18-day-old pregnant SD rats). ${ }^{25}$ The meninges and blood vessels were removed from the brain and then brain tissues were digested with $0.25 \%$ trypsin (with EDTA) for 5 min at $37^{\circ} \mathrm{C}$. The tissues were washed three times with PBS to terminate trypsin digestion. Then, brain tissue suspensions were centrifuged at 1500 r.p.m. for $5 \mathrm{~min}$, and the cells were suspended in a Neurobasal-A medium containing 2\% B27, 2 mu L-glutamine,
$50 \mathrm{U} / \mathrm{ml}$ penicillin and $50 \mathrm{U} / \mathrm{ml}$ streptomycin (all from Gibco, Carlsbad, CA, USA). Finally, cells were plated in 6- or 12-well plates in a fresh medium and later half the medium was changed with fresh medium every 2 days.

For primary microglial cultures, the whole brains of 1 - day-old rats were used. ${ }^{26}$ The digestion was similar to neurons, but the pellets after centrifugation were suspended in DMEM/F12 containing $10 \%$ fetal bovine serum, $1 \mathrm{~mm}$ sodium pyruvate, $2 \mathrm{~mm}$ L-glutamine, $100 \mathrm{mM}$ nonessential amino acids, $50 \mathrm{U} / \mathrm{ml}$ penicillin and $50 \mathrm{U} / \mathrm{ml}$ streptomycin (all from Gibco). Then, cells were seeded into $150 \mathrm{~cm}^{2}$ culture flask in a fresh medium and half the medium was changed with fresh medium every 2 days. Two weeks after initial seeding, a confluent polylayer of glial cells could be observed.

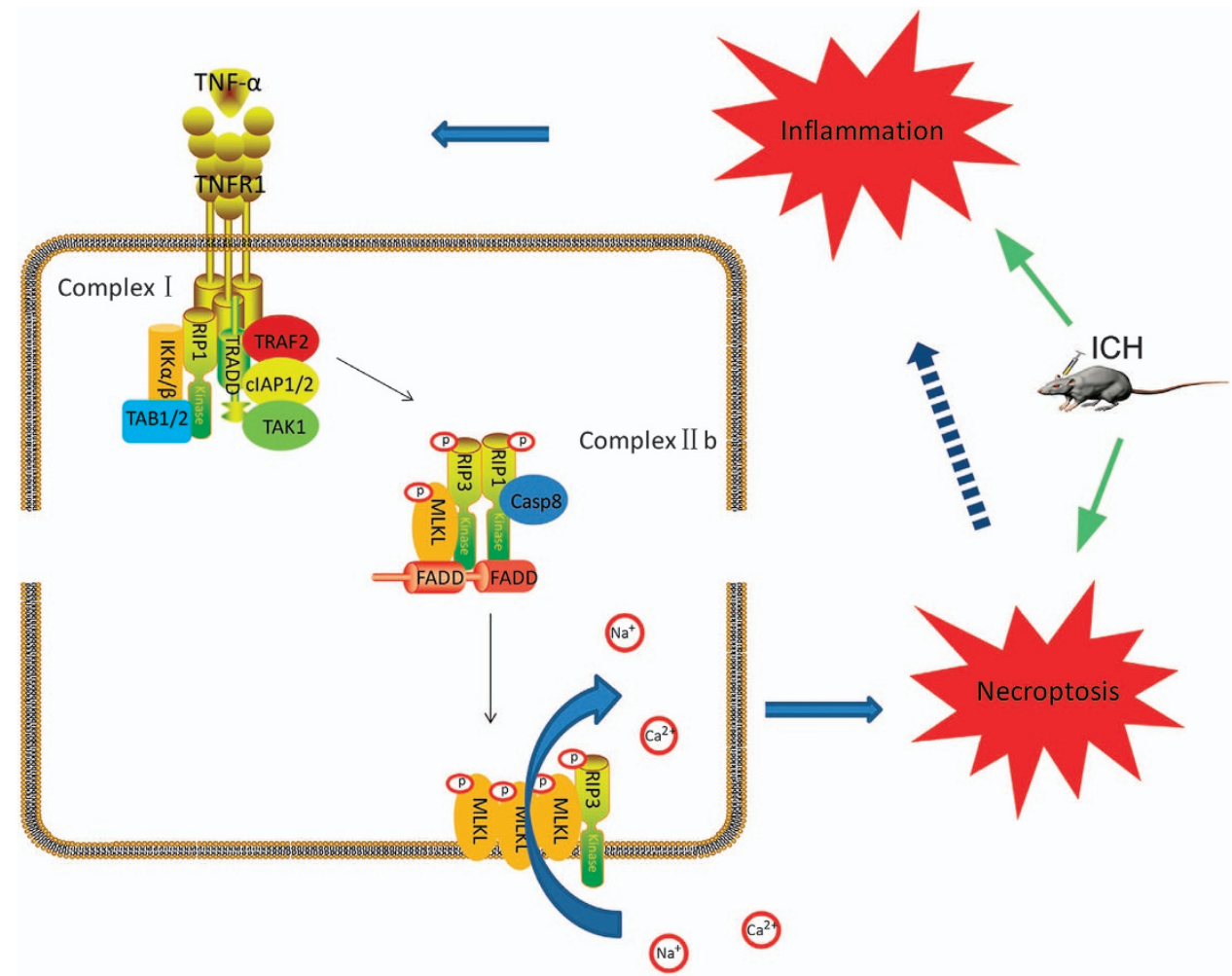

Figure 6 Hypothesized model for molecular mechanism involved in necroptosis in brain tissues after ICH. After ICH occurs, microglia were rapidly activated and released an amount of inflammatory cytokines such as TNF- $\alpha$. TNFR1 can spontaneously trimerize at the plasma membrane. When TNF- $\alpha$ binds to TNFR1, the conformation of these receptor trimers would be changed, allowing their cytosolic tails recruit multiple proteins, and then develop a complex (complex I) including TRADD (TNF- $\alpha$ receptor-associated death domain), RIP1, TRAF2 (TNFR associated factor 2), CIAP1 and CIAP2 (cellular inhibitor of apoptosis 1/2). Deubiquitylation of RIP1 mediates its transition from complex I to complex II, and then cooperates with RIP3 for recruitment of MLKL (mixed lineage kinase domain-like protein), FADD (FAS-associated protein with a death domain) and caspase8. In this complex Ilb, RIP1 and RIP3 are inhibited by caspase-8. When caspase-8 is inactive, complex Illb would carry out the TNF- $\alpha$-mediated necroptotic pathway. Further, complex of MLKL and RIP3 transfers to the cell membrane and forms a channel, and then the internal flow of $\mathrm{Ca}^{2+}$ or $\mathrm{Na}^{+}$is caused. Finally, the cell is dead by the necroptotic pathway. As necroptosis can further promote inflammation, it suggests that it can possibly have a positive feedback relationship between necroptosis and inflammation in brain injury after $\mathrm{ICH}$

Figure 5 Phosphorylation of RIP1 switched necroptosis in cultured neurons in model of ICH in vitro. As phosphorylation of RIP1 has an essential role of necroptosis, we used overexpression and with a mutation of phosphorylation site (S166A) of RIP1 to investigate its role in necroptosis in ICH. (a) Flow cytometry indicated that, compared with the conditioned medium group, it turned up a higher ratio of necroptosis ( 66.7\%) and a lower ratio of apoptosis ( 0.9\%) in neurons during overexpression of RIP1 by Ad-RIP1 transfection. Besides, this phenomenon could be significantly reduced in the $\mathrm{S} 166 \mathrm{~A}$ group (necroptosis $\sim 34.2 \%$ ) and in the Nec-1 treatment group (necroptosis $~ 21.7 \%$ ). (b) Related bar graph showed four different conditions of neurons that had been stated above. ${ }^{*} \mathrm{P}=0.0001 \mathrm{in} \mathrm{Pl}+/ \mathrm{Annexin} \mathrm{V}-$ cells versus the conditioned medium group; ${ }^{2} \& P=0.0027$ in $\mathrm{PI}+/$ Annexin $\mathrm{V}$ - cells versus the Ad-RIP1 group; ${ }^{\$} P<0.0001$ in $\mathrm{Pl}+/$ Annexin $\mathrm{V}-$ cells and ${ }^{\$ \$} P<0.0001$ in $\mathrm{PI}-/$ Annexin $\mathrm{V}+$ cells versus the Ad-RIP1 group; all were unpaired t-test, $n=3$. (c) IP demonstrated that interactions of RIP1 and RIP3, RIP1 and MLKL, and RIP1 and caspase-8 were increased in the Ad-RIP1 group. These increased interactions could be attenuated remarkably in the S166A group. (d) Consistent data analysis of IP. ${ }^{\star *} P=0.0015$ in $p-S e r$, ${ }^{\star *} P=0.0004$ in RIP3, ${ }^{* \star} P=0.0013$ in MLKL and ${ }^{* *} P=0.0003$ in caspase-8 versus the conditioned medium group; ${ }^{\&} P=0.0493$ in $p-S e r,{ }^{\&} P=0.0126$ in RIP3, ${ }^{\text {\& }} P=0.0036$ in MLKL and ${ }^{\text {\&\& }} P=0.0018$ in caspase-8 versus the Ad-RIP1 group. The mean values for the control group were normalized to 1.0; all were unpaired $t$-test, $n=3$. (e) Pl and Hoechst staining indicated that the ratio of necroptosis upregulated (as arrows point to, $\mathrm{Pl}+/$ Hoechst + cells) in the overexpression group, whereas mutation of the phosphorylation site inhibited this effect. Scale bar $=50 \mu \mathrm{m}$. (f) Numbers of $\mathrm{Pl}+/$ Hoechst+ cells, ${ }^{\star}{ }^{\star} P<0.0001$ versus the conditioned medium group; ${ }^{\& \&} P<0.0001$ versus Ad-RIP1 group; all were unpaired $t$-test, $n=6$. All data are expressed as means \pm S.E.M. 
a Experiment 1

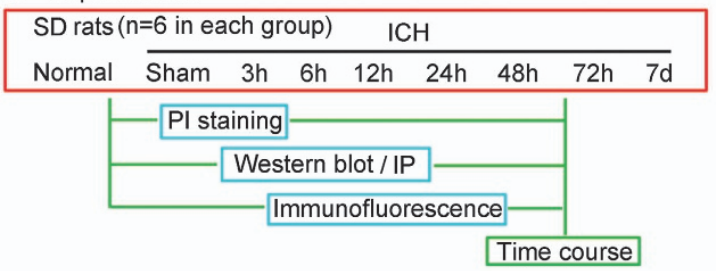

b Experiment 2

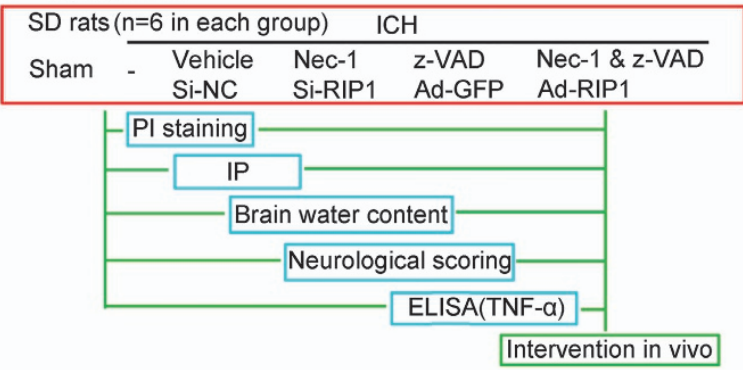

C Experiment 3

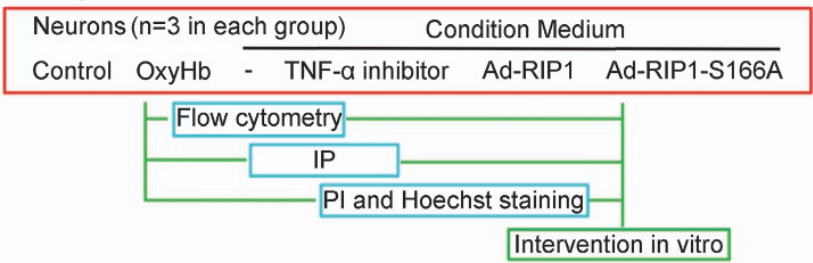

Figure 7 Experimental designs. (a) Experiment 1 was designed to show the time course of RIP1 expression after ICH and to determine a time point for the next experiment. (b) Experiment 2 was designed to explore the roles of RIP1 and necroptosis in brain injury after ICH in vivo. (d) Experiment 3 was designed to study the potential mechanism of RIP1 and necroptosis in brain injury after ICH in vitro.

Table 2 Neurobehavioral evaluation

\begin{tabular}{llc}
\hline Category & Behavior & Score \\
\hline Appetite & Finished meal & 0 \\
& Left meal unfinished & 1 \\
& Scarcely ate & 2 \\
\multirow{4}{*}{ Activity } & Walk and reach at least three corners of the cage & 0 \\
& Walk with some stimulations & 1 \\
& Almost always lying down & 2 \\
Deficits & No deficits & 0 \\
& Unstable walk & 1 \\
& Impossible to walk & 2 \\
\hline
\end{tabular}

The microglia was separated from astrocytes by shaking the flask at 150 r.p.m. for $4 \mathrm{~h}$, and then it was collected by centrifugation and reseeded in 12-well plates with fresh medium.

Drug administration. Nec-1 was prepared in DMSO at a concentration of $1 \mu \mathrm{g} / 3 \mu \mathrm{l}^{27}$ and z-VAD (all from Santa Cruz Biotechnology, Santa Cruz, CA, USA) was prepared in DMSO at a concentration of $100 \mu \mathrm{m} .{ }^{10}$ At $1-2 \mathrm{~h}$ before $\mathrm{ICH}$, both the inhibitors were injected into the lateral cerebral ventricle at a volume of $3 \mu \mathrm{l}$. Equal volumes of DMSO were used as vehicle. For in vitro experiments, the TNF- $\alpha$ inhibitor (Santa Cruz Biotechnology) was also dissolved in DMSO at a final concentration of $50 \mu \mathrm{M}$ in a neuronal medium. The final concentration of Nec-1 and $\mathrm{z}$-VAD was 30 and $100 \mu \mathrm{M}$, respectively, in a neuronal medium.
$\mathrm{ICH}$ models in vitro. An $\mathrm{ICH}$ model in vitro was established by neuronal stimulation using $\mathrm{OxyHb} .{ }^{21}$ Neurons were treated with $\mathrm{OxyHb}(10 \mu \mathrm{M})$ for $6 \mathrm{~h}$ at $37^{\circ} \mathrm{C}$ in $5 \% \mathrm{CO}_{2}$, and then cell medium was removed, washed three times with PBS and followed by other experiments.

The other in vitro $\mathrm{ICH}$ model used conditioned medium to treat neurons. First, microglia were treated with $\mathrm{OxyHb}(10 \mu \mathrm{M})$ and then incubated for $24 \mathrm{~h}$ at $37^{\circ} \mathrm{C}$ with $5 \% \mathrm{CO}_{2}$. The supernatant collected was centrifuged at 10000 r.p.m. for $5 \mathrm{~min}$ and then was transferred to a conditioned medium. Half of the neuronal medium was changed with conditioned medium and incubated for another $6 \mathrm{~h}$ at $37^{\circ} \mathrm{C}$ with $5 \%$ $\mathrm{CO}_{2}$. The cell medium was removed, washed three times with PBS and followed by other experiments.

Transfection of siRNA and adenoviruses in vivo and in vitro. The following three kinds of recombinant adenoviruses were used: (1) adenoviruses containing rat RIP1 (Ad-RIP1; Genbank ID: 157824040) that was used to overexpress RIP1 protein; (2) adenovirus with rat RIP1 with mutation of S166A (Ser166 of RIP1 was mutated to alanine, Ad-RIP1-S166A); and (3) adenovirus with human GFP (AdGFP) as a control to Ad-RIP1. Ad-RIP1 and Ad-RIP1-S166A (both $6 \times 10^{9} \mathrm{PFU} / \mathrm{ml}$ ) and Ad-GFP $\left(2 \times 10^{9} \mathrm{PFU} / \mathrm{ml}\right)$ were produced by Genescript (Nanjing, China). All of them were stored at $-80^{\circ} \mathrm{C}$ and diluted to $1 \times 10^{9} \mathrm{PFU} / \mathrm{ml}$ in an enhanced transfection solution (Genescript) before intracerebroventricular injection in vivo and diluted to $1 \times 10^{8} \mathrm{PFU} / \mathrm{ml}$ before being transfected to the cultured neuron.

The following two kinds of siRNAs were used: (1) disorganizing rat RIP1 mRNA (Si-RIP1) to silence its transcription and (2) scramble siRNA (Si-negative control) (both from Genescript). According to the manufacturer's instructions for Entransterin vivo RNA transfection reagent (Engreen, Shanghai, China), 500 pmol RIP1 siRNA and $500 \mathrm{pmol}$ scramble siRNA were dissolved in $5 \mu \mathrm{l}$ RNase-free water. Then, $10 \mu \mathrm{l}$ Entranster-in vivo RNA transfection reagent was added to $5 \mu \mathrm{l}$ siRNA or $5 \mu \mathrm{l}$ scramble siRNA. After mixing for another $15 \mathrm{~min}$, Entranster-in vivo-siRNA mixture was injected intracerebroventricularly $24 \mathrm{~h}$ before ICH. RIP1 siRNA sequences were 
as follows: sense, 5'-GGAACAACGGAGUAUAUAAdTdT-3' and Antisense: $3^{\prime}$-dTdTCCUUGUUGCCUCAUAUAUU-5'.

Western blot analysis. Western blot analysis was performed as indicated previously. ${ }^{25}$ Briefly, the brain samples or extracted cells were mechanically lysed in RIPA lysis buffer (Beyotime, Shanghai, China). Then we used enhanced BCA Protein Assay Kit (Beyotime) to measure protein concentrations by the bicinchoninic acid method. The protein samples (50 $\mu \mathrm{g}$ per lane) were then loaded onto a $10 \%$ SDS-polyacrylamide gel, separated and electrophoretically transferred to a polyvinylidene difluoride membrane (Millipore Corporation, Billerica, MA, USA), which was then blocked with $5 \%$ bovine serum albumin (BioSharp, Anhui, China) ( $1 \mathrm{~h}$ at room temperature). Then, the membrane was incubated for $12 \mathrm{~h}$ at $4{ }^{\circ} \mathrm{C}$ with primary antibodies. The primary antibodies used were RIP1, RIP3, MLKL, caspase8, p-Ser (all from Santa Cruz Biotechnology) and albumin (Abcam, Cambridge, UK; ab106582). Besides, the $\beta$-tubulin was also detected and served as a loading control. Later, the membrane was incubated with related HRP-conjugated secondary antibody (Santa Cruz Biotechnology) for $2 \mathrm{~h}$ at room temperature. We revealed the band signals via the Enhanced Chemiluminescence (ECL) Kit (Beyotime), and the relative quantity of proteins was analyzed via the Image $\mathrm{J}$ Software (NIH, Bethesda, MD, USA) and normalized to that of the loading control as discussed previously. In addition, the levels of phosphorylation were evaluated as the ratio of phosphoprotein to total protein.

Immunoprecipitation. Briefly, according to our previous report, ${ }^{25}$ the brain samples were lysed in ice-cold RIPA lysis buffer (Beyotime). For IP, the lysate was incubated with specific antibodies against RIP1 or rabbit lgG (negative control) overnight at $4{ }^{\circ} \mathrm{C}$ with agitation. Protein $\mathrm{A}+\mathrm{G}$ Sepharose beads were then added to each immune complex, and the lysate-bead mixture was incubated for $4 \mathrm{~h}$ at $4{ }^{\circ} \mathrm{C}$ under rotary agitation. SDS-PAGE and immunoblotting were then performed for further protein separation and detection.

Immunofluorescent staining. The brain samples were fixed in $4 \%$ paraformaldehyde, embedded in paraffin and then cut into $4 \mu \mathrm{m}$ sections. The cultured neurons were fixed in $4 \%$ paraformaldehyde. Then, the sections and neurons were incubated with primary antibody against RIP1, RIP3, MLKL and caspase-8 and secondary antibodies. Normal rabbit lgG, normal mouse IgG and normal goat IgG were used as negative controls for the immunofluorescence assay (data not shown). Nuclei were stained with DAPI mounting medium. Finally, the sections and neurons were observed in a fluorescence microscope (OLYMPUS BX50/BX-FLA/DP70; Olympus Co., Tokyo, Japan). The relative fluorescence intensity was analyzed with the Image $\mathrm{J}$ program. The quantitative analysis was performed by an observer who was blinded to the experimental group.

PI and TUNEL staining. For all experiments, PI (Sigma-Aldrich, St. Louis, MO, USA) was administered intraperitoneally $(1 \mu \mathrm{g} / \mathrm{g})$ while the $\mathrm{ICH}$ model was established. ${ }^{28}$ According to the manufacturer's protocol (Roche, Mannheim, Germany), we detected cell apoptosis via TUNEL staining. The brain was removed and frozen in liquid nitrogen and then stored at $-80^{\circ} \mathrm{C}$. Then, brain sections $(12 \mu \mathrm{m})$ were cut on a cryostat 150 to $200 \mu \mathrm{m}$ apart along the anterior-posterior lesion and were placed on poly-L-lysine-coated glass slides $\left(-80^{\circ} \mathrm{C}\right)$. Next, the sections were incubated with TUNEL staining $\left(37^{\circ} \mathrm{C}\right.$ for $\left.1 \mathrm{~h}\right)$. After washing three times with PBST, sections were visualized by a fluorescence microscope (OLYMPUS BX50/BX-FLA/ DP70; Olympus $\mathrm{C}_{0}$.). In conclusion, PI/TUNEL+ cells were counted by observers blinded to the groups of experiment. To evaluate necropoptosis/apoptosis of cells, we examined and photographed six microscopic fields per sample and the index was defined as the average number in each section. As reported previously, $\mathrm{Pl}+/ \mathrm{TUNEL}-$ is defined as the pure necropoptosis, $\mathrm{PI}-/ T U N E L+$ is defined as pure apoptosis, and $\mathrm{Pl}+$ TUNEL+ is defined as the mixed cell death. ${ }^{29}$

Brain edema and BBB injury. As described previously, ${ }^{21}$ the wet-dry method was adopted to evaluate the index of brain edema and BBB injury. Briefly, after brain tissues were removed and collected, the samples were weighed immediately (wet weight), dried $\left(100^{\circ} \mathrm{C}\right.$ for $72 \mathrm{~h}$ ) and then reweighed (dry weight). Thus, brain edema was calculated as ((wet weight-dry weight)/wet weight) $\times$ $100 \%$. Meanwhile, the level of albumin in brain tissues was regarded as the index of BBB injury. The western blot was used to test the level of albumin.

Neurobehavioral evaluation. At $24 \mathrm{~h}$ after $\mathrm{ICH}$, all the rats in the experiments were examined for behavioral impairment using a scoring system and were monitored for appetite, activity and neurological defects, as reported previously ${ }^{30}$ (for more details see Table 2 ).

Assay of inflammatory cytokines (TNF- $\boldsymbol{a}$ ). We used specific Rat Tumor necrosis factor $\alpha$, TNF- $\alpha$ ELISA KIT (Bio-Swamp; Hubei, China) to quantify the levels of TNF- $\alpha$ in the CSF according to the manufacturer's instructions.

Annexin $\mathrm{V}$ and $\mathrm{PI}$ staining in vitro. After various treatments, neurons were trypsinized by $0.25 \%$ trypsin (without EDTA) and centrifuged at 1500 r.p.m. for $5 \mathrm{~min}$, and the resulting cell pellet was resuspended in $500 \mu \mathrm{l}$ binding buffer. Later, $5 \mu \mathrm{l}$ Annexin V and $5 \mu \mathrm{l}$ PI (Beyotime, Shanghai, China) were added to the cell suspension. After $20 \mathrm{~min}$ of incubation at $37^{\circ} \mathrm{C}$ in the dark, the cells were analyzed by flow cytometry (FACS Cabibur; BD, San Diego, CA, USA) and at least 20000 events per sample were recorded.

PI and Hoechst staining in vitro. After various treatments, add $5 \mu$ Hoechst and $5 \mu \mathrm{l} \mathrm{PI}$ (Beyotime, Shanghai, China) to the cell medium. After $20 \mathrm{~min}$ of incubation at $4{ }^{\circ} \mathrm{C}$ in the dark, the cells were analyzed by a fluorescence microscope (OLYMPUS BX50/BX-FLA/DP70; Olympus Co., Tokyo, Japan.)

Statistical analysis. All data are presented as mean \pm S.E.M. GraphPad Prism 5.0 software (GraphPad, San Diego, CA, USA) was used for statistical analysis. Data sets were tested for normality of distribution with KolmogorovSmirnov test. Data groups (two groups) with normal distribution were compared using two-sided unpaired Student's $t$-test, and the Mann-Whitney U-test was used for nonparametric data. $P<0.05$ indicates a statistically significant difference.

\section{Conflict of Interest}

The authors declare no conflict of interest.

Acknowledgements. This work was supported by Suzhou Key Medical Center (Szzx201501), grants from the National Natural Science Foundation of China (No. 81601007), Scientific Department of Jiangsu Province (No. BL2014045), Education Department of Jiangsu Province (No. 16KJB320008), Suzhou Government (No. SZS201413 and SYS201608) and a project funded by the Priority Academic Program Development of Jiangsu Higher Education Institutions.

1. Joseph MJ, Caliaperumal J, Schlichter LC. after intracerebral hemorrhage, oligodendrocyte precursors proliferate and differentiate inside white-matter tracts in the rat striatum. Trans Stroke Res 2016; 7: 192-208.

2. Behrouz R. Re-exploring tumor necrosis factor alpha as a target for therapy in intracerebral hemorrhage. Transl Stroke Res 2016; 7: 93-96.

3. Jiang B, Li L, Chen Q, Tao Y, Yang L, Zhang B et al. Role of glibenclamide in brain injury after intracerebral hemorrhage. Transl Stroke Res 2016; 10.1007/s12975-016-0506-2.

4. Dang G, Yang Y, Wu G, Hua Y, Keep RF, Xi G. Early erythrolysis in the hematoma after experimental intracerebral hemorrhage. Trans/ Stroke Res 2016; 10.1007/s12975-0160505-3.

5. Sukumari-Ramesh S, Alleyne CH Jr, Dhandapani KM. The histone deacetylase inhibitor suberoylanilide hydroxamic acid (SAHA) confers acute neuroprotection after intracerebral hemorrhage in mice. Transl Stroke Res 2016; 7: 141-148.

6. Elliott J, Smith M. The acute management of intracerebral hemorrhage: a clinical review. Anesth Analg 2010; 110: 1419-1427.

7. Aronowski J, Zhao X. Molecular pathophysiology of cerebral hemorrhage: secondary brain injury. Stroke 2011; 42: 1781-1786.

8. Zong WX, Thompson CB. Necrotic death as a cell fate. Genes Dev 2006; 20: 1-15.

9. Golstein P, Kroemer G. Cell death by necrosis: towards a molecular definition. Trends Biochem Sci 2007; 32: 37-43.

10. Degterev A, Huang Z, Boyce M, Li Y, Jagtap P, Mizushima N et al. Chemical inhibitor of nonapoptotic cell death with therapeutic potential for ischemic brain injury. Nat Chem Biol 2005; 1: 112-119.

11. Galluzzi L, Kepp O, Krautwald S, Kroemer G, Linkermann A. Molecular mechanisms of regulated necrosis. Semin Cell Dev Biol 2014; 35: 24-32.

12. Nikoletopoulou V, Markaki M, Palikaras K, Tavernarakis N. Crosstalk between apoptosis necrosis and autophagy. Biochim Biophys Acta 2013; 1833: 3448-3459.

13. Nguyen HX, O'Barr TJ, Anderson AJ. Polymorphonuclear leukocytes promote neurotoxicity through release of matrix metalloproteinases, reactive oxygen species, and TNF- $\alpha$. J Neurochem 2007; 102: 900-912.

14. Lo EH, Dalkara T, Moskowitz MA. Mechanisms, challenges and opportunities in stroke. Nat Rev Neurosci 2003; 4: 399-415. 
15. Jouan-Lanhouet S, Riquet F, Duprez L, Vanden Berghe T, Takahashi N, Vandenabeele P. Necroptosis, in vivo detection in experimental disease models. Semin Cell Dev Biol 2014; 35: 2-13.

16. Delgado $P$, Cuadrado E, Rosell A, Alvarez-Sabin J, Ortega-Aznar A, Hernandez-Guillamon M et al. Fas system activation in perihematomal areas after spontaneous intracerebral hemorrhage. Stroke 2008; 39: 1730-1734.

17. Ke K, Rui Y, Li L, Zheng H, Xu W, Tan X et al. Upregulation of EHD2 after intracerebra hemorrhage in adult rats. $J$ Mol Neurosci 2014; 54: 171-180.

18. Kono H, Rock KL. How dying cells alert the immune system to danger. Nat Rev Immunol 2008; 8: 279-289.

19. Su X, Wang H, Kang D, Zhu J, Sun $Q$, Li T et al. Necrostatin-1 ameliorates intracerebral hemorrhage-induced brain injury in mice through inhibiting RIP1/RIP3 pathway. Neurochem Res 2015; 40: 643-650.

20. King MD, Whitaker-Lea WA, Campbell JM, Alleyne CH Jr, Dhandapani KM. Necrostatin-1 reduces neurovascular injury after intracerebral hemorrhage. Int J Cell Biol 2014; 2014: 495817.

21. Zhai W, Chen D, Shen H, Chen Z, Li H, Yu Z et al. A1 adenosine receptor attenuates intracerebral hemorrhage-induced secondary brain injury in rats by activating the P38MAPKAP2-Hsp27 pathway. Mol Brain 2016; 9: 66

22. Gao Z, Wang J, Thiex R, Rogove AD, Heppner FL, Tsirka SE. Microglial activation and intracerebral hemorrhage. Acta Neurochir Suppl 2008; 105: 51-53.

23. Lei B, Dawson HN, Roulhac-Wilson B, Wang H, Laskowitz DT, James ML. Tumor necrosis factor alpha antagonism improves neurological recovery in murine intracerebral hemorrhage. J Neuroinflamm 2013; 10: 103

24. Deinsberger W, Vogel J, Kuschinsky W, Auer LM, Boker DK. Experimental intracerebra hemorrhage: description of a double injection model in rats. Neurol Res 1996; 18: 475-477.

25. Shen $\mathrm{H}$, Chen Z, Wang Y, Gao A, Li H, Cui Y et al. Role of neurexin-1 beta and neuroligin-1 in cognitive dysfunction after subarachnoid hemorrhage in rats. Stroke 2015; 46: 2607-2615

26. Liu B, Wang K, Gao HM, Mandavilli B, Wang JY, Hong JS. Molecular consequences of activated microglia in the brain: overactivation induces apoptosis. J Neurochem 2001; 77: 182-189.
27. Yin B, Xu Y, Wei RL, He F, Luo BY, Wang JY. Inhibition of receptor-interacting protein 3 upregulation and nuclear translocation involved in necrostatin-1 protection against hippocampal neuronal programmed necrosis induced by ischemia/reperfusion injury. Brain Res 2015; 1609: 63-71.

28. Ding W, Shang L, Huang JF, Li N, Chen D, Xue LX et al. Receptor interacting protein 3-induced RGC-5 cell necroptosis following oxygen glucose deprivation. BMC Neurosci 2015; 16: 49

29. Zhu X, Tao L, Tejima-Mandeville E, Qiu J, Park J, Garber K et al. Plasmalemma permeability and necrotic cell death phenotypes after intracerebral hemorrhage in mice. Stroke 2012; 43: 524-531.

30. Ma J, Wang Z, Liu C, Shen H, Chen Z, Yin J et al. Pramipexole-Induced hypothermia reduces early brain injury via PI3K/AKT/GSK3beta pathway in subarachnoid hemorrhage rats. Scientific Rep 2016; 6: 23817.

(c) Cell Death and Disease is an open-access journal published by Nature Publishing Group. This work is licensed under a Creative Commons Attribution 4.0 International License. The images or other third party material in this article are included in the article's Creative Commons license, unless indicated otherwise in the credit line; if the material is not included under the Creative Commons license, users will need to obtain permission from the license holder to reproduce the material. To view a copy of this license, visit http://creativecommons.org/licenses/by/4.0/

(C) The Author(s) 2017 